\title{
Exceptional stability of a perilipin on lipid droplets depends on its polar residues, suggesting multimeric assembly
}

\author{
Manuel Giménez-Andrés ${ }^{1,2}$, Tadej Emeršič ${ }^{3}$, Sandra Antoine-Bally ${ }^{1}$, Bruno Antonny ${ }^{4}$, Jure \\ Derganc $^{3,5}$, Alenka Čopič1,6
}

1. Institut Jacques Monod, CNRS, UMR 7592, Université de Paris, France

2. Université Paris-Saclay, 91405, Orsay, France

3. Institute of Biophysics, Faculty of Medicine, University of Ljubljana

4. Université Côte d'Azur, CNRS, IPMC, 06560 Valbonne, France

5. Chair of Microprocess Engineering and Technology - COMPETE, University of Ljubljana

6. Corresponding author: alenka.copic@ijm.fr

\section{Abstract}

Numerous proteins target lipid droplets (LDs) through amphipathic helices (AHs). It is generally assumed that AHs insert bulky hydrophobic residues in packing defects at the LD surface. However, this model does not explain the targeting of perilipins, the most abundant and specific amphipathic proteins of LDs. The gigantic Plin4 contains a highly repetitive $\mathrm{AH}$ that lacks bulky hydrophobic residues, and its LD targeting depends strongly on its length. We show that Plin4 forms a remarkably immobile protein layer at the surface of cellular or artificial LDs, making them stable over days. This Plin4 AH feature is not shared with the AHs of other perilipins, which display much faster dynamics on lipid surfaces. Plin4 AH stability on LDs is exquisitely sensitive to the nature and distribution of its polar residues. These results suggest that Plin4 forms stable arrangements of adjacent AHs via polar interactions. 


\section{Introduction}

Lipid droplets (LDs) are cellular organelles specialized for storage of lipids and maintenance of cellular lipid homeostasis. They are composed of a neutral lipid core that is covered by a monolayer of phospholipids and other amphiphilic lipids, and by proteins (Thiam et al., 2013b; Olzmann and Carvalho, 2019). LDs vary in size over four orders of magnitude, depending on organism/cell type and fasting state of a cell; in mature adipocytes, the majority of the cell can be occupied by a single LD measuring $>100 \mu \mathrm{m}$ in diameter (Lundquist et al., 2020; Stenkula and Erlanson-Albertsson, 2018). Secreted lipoprotein particles are similar to LDs in terms of their over-all composition but are much smaller, ranging from 10 to $1000 \mathrm{~nm}$ in diameter (Ohsaki et al., 2014).

Different types of proteins have been found to associate with LDs (Brasaemle et al., 2004; Bersuker et al., 2018; Kory et al., 2015; Pataki et al., 2018; Mejhert et al., 2020). They can be either stably embedded in the LD monolayer, coming by diffusion from the endoplasmic reticulum (ER), from which LDs emerge, or they associate with LDs peripherally from the cytosol (Ohsaki et al., 2014; Bersuker and Olzmann, 2017). Many of them are enzymes involved in lipid synthesis or hydrolysis, for example triglyceride synthases, acyltransferases, lipases and their inhibitors or activators (Wilfling et al., 2013; Zechner et al., 2017). Proteins can also regulate LDs in a non-enzymatic manner. A prominent example is the perilipins: in mammals, this is a family of five proteins that share related structural features and are abundant on LDs (Sztalryd and Brasaemle, 2017). They vary in their tissue distribution: Plin2 and Plin3 are widely expressed, whereas Plin1 and Plin4 are most highly expressed in adipocytes, and Plin5 is enriched in oxidative tissues (Wolins et al., 2006; Brasaemle et al., 2004; Wolins et al., 2003). Plin4 and Plin5 are also enriched in muscle tissues. Less closely related abundant LD proteins have been identified in many other species (Gao et al., 2017; Granneman et al., 2017; Miura et al., 2002). Whereas perilipins contain no known enzymatic motifs, a number of them, in particular Plin1, have been shown to regulate the recruitment of lipases to the LD surface (Sztalryd and Brasaemle, 2017).

Many LD-localized proteins use amphipathic helices (AHs) to directly interact with the LD lipid surface (Bersuker and Olzmann, 2017; Giménez-Andrés et al., 2018). All mammalian perilipins 
contain a predicted $\mathrm{AH}$ region in their $\mathrm{N}$-terminal part, which has been shown to be important for their LD localization (McManaman et al., 2003; Nakamura and Fujimoto, 2003; Bulankina et al., 2009; Rowe et al., 2016; Copic et al., 2018). This region is composed of 11-aa repeats that would fold into a 3-11 helix, which has been structurally characterized in $\alpha$-synuclein and is slightly more extended than the classical $\alpha$-helix (Bussell and Eliezer, 2003; Jao et al., 2008). Other regions, including a C-terminal region that can fold into a 4-helix bundle (Hickenbottom et al., 2004), can also contribute to LD targeting to varying extents (Targett-Adams et al., 2003; Subramanian et al., 2004; Nakamura et al., 2004; Ajjaji et al., 2019). Interestingly, both the 11aa repeat region and the 4-helix bundle bear structural similarities with apolipoproteins, which are required for formation of lipoprotein particles (Saito et al., 2003; Melchior et al., 2017).

The 11-aa repeat $\mathrm{AH}$ region is by far the longest in Plin4, containing close to 1000 aa in the human protein, with repeats that are highly homologous at the 33-aa level (Copic et al., 2018; Scherer et al., 1998). The aa composition of Plin4 AH reveals a striking bias towards small hydrophobic residues, in particular $\mathrm{V}, \mathrm{T}$ and $\mathrm{A}$, whereas large residues such as $\mathrm{W}$ and $\mathrm{F}$ are almost entirely absent (Figure 1A). Whereas the 11-aa repeat regions of other perilipins are about 10-times shorter than that of Plin4 and the repeats are far less conserved, they are similar in terms of their over-all aa composition. We have demonstrated that the Plin4 $\mathrm{AH}$ region is unfolded in solution, but adopts a highly helical structure in contact with a lipid surface. The low hydrophobicity of this $\mathrm{AH}$ promotes specific targeting to LDs, which are permissive for the binding of many amphipathic proteins (Copic et al., 2018; Prévost et al., 2018). This is likely due to the physical properties of the LD surface, where the spreading of the phospholipid monolayer leads to exposure of the hydrophobic core with which the hydrophobic face of an AH can interact more strongly (Bacle et al., 2017; Chorlay et al., 2019). Due to its extreme length, Plin4 in particular can cover a large LD surface and could act as a substitute for phospholipids (Copic et al., 2018). A recent study has identified expansion of Plin4 33-aa repeats in a family with a rare autosomal dominant progressive myopathy, underscoring the importance of studying this protein (Ruggieri et al., 2020).

Due to their high abundance on LDs, perilipins are often referred to as LD coat proteins (Sztalryd and Brasaemle, 2017). Protein coats have been well characterized on transport 
vesicles, for example COPI, COPII and clathrin coat. In all these cases, the coat forms in a tightly controlled manner by sequential recruitment of coat subunits on the membrane surface (Schekman and Orci, 1996; Taylor et al., 2011). Importantly, coat subunits laterally interact to form a highly polymerized structure covering the surface of a vesicle (Faini et al., 2013). Coat polymerization is in fact the main force that generates these membrane vesicles (Saleem et al., 2015). Perilipins have not been shown to be directly involved in LD budding from the ER; LD formation may be principally driven by lipids, with proteins playing a more regulatory role (Ben M'barek et al., 2017; Chorlay et al., 2019; Santinho et al., 2020). On the other hand, COPI coat components have also been observed to bind to and influence LDs and to regulate recruitment of other LD proteins (Bouvet et al., 2013; Guo et al., 2008; Thiam et al., 2013a; Wilfling et al., 2014; Soni et al., 2009).

Here, we ask whether perilipins possess any of the qualities traditionally associated with protein coats. We focus on the 11-aa repeat $\mathrm{AH}$ regions of mammalian perilipins, which directly associate with the lipid surface of LDs. We analyze the stability of perilipin AHs on the lipid surface and their ability to form an immobile structure using various cellular and biochemical approaches, as well as a novel microfluidics set-up to follow the interaction of AHs with oil over time. We show that one perilipin, Plin4, is capable of making highly stable protein-lipid structures by forming an immobile coat on the surface of pure oil using its uniquely long $\mathrm{AH}$. These protein-oil droplets remain stable over the course of many days. In contrast, the interaction of the AHs from other perilipins with LDs or with oil is highly dynamic. Extensive mutagenesis shows that the $\mathrm{AH}$ of Plin4 can form an immobile coat due to its organized structure that could enable interhelical interactions on the lipid surface. The precise organization of Plin4 $\mathrm{AH}$, along with its variable length, are promoted during mammalian evolution, suggesting an important role for this protein in mammals. 


\section{Results}

\section{Purified Plin4 AH forms very stable protein-oil emulsions}

We have previously shown that the AH of Plin4 is optimized for LD binding both by its length and particular aa composition (Copic et al., 2018). The AH sequence of human Plin4 is composed of $\sim 29$ highly homologous 33-aa repeats (Figure 1A). The efficiency of LD targeting correlated with $\mathrm{AH}$ length: at least four 33-aa repeats of the wild-type Plin4 $\mathrm{AH}$ were needed to detect some LD localization in HeLa cells (Figure 1B). Furthermore, the strong bias towards small residues is decisive for LD targeting: mutations that increased hydrophobicity $(T>V)$ made Plin4 promiscuous for other organelles besides LDs; mutations that decreased hydrophobicity $(\mathrm{T}>\mathrm{S})$ rendered Plin4 cytosolic (Figure 1B).

The amphipathic region of Plin4 is capable of emulsifying triolein upon vigorous mechanical mixing (vortex) in aqueous buffer in the absence of any other surfactant, such as phospholipids. A similar result was obtained with a shorter Plin4 AH construct containing 4 33-aa repeats (Plin4 4mer) or a longer construct comprising 12 33-aa repeats (Plin4 12mer) (Figure 1C) (Copic et al., 2018). Electron microscopy and dynamic light scattering (DLS) revealed that emulsions of triolein and Plin4 12mer contained spherical oil particles with a large range of sizes; typically with a diameter of 50 to $500 \mathrm{~nm}$ (mean $\approx 200 \mathrm{~nm}$ ), although some larger particles (diameter $>1 \mu \mathrm{m}$ ) could also be observed (Copic et al., 2018). We focused on these latter particles, whose size made them suitable for imaging by fluorescence light microscopy. For this, we performed triolein emulsification in the presence Plin4 $12 \mathrm{mer}$ labeled with the fluorescent dye Alexa488 (Plin4 12mer-A488), mixed with unlabeled Plin4 12 mer. The spherical particles displayed a homogenous fluorescent surface, which allowed us to perform dynamics measurements by fluorescence recovery after photobleaching (FRAP) (Figure 1D).

In the first FRAP protocol, we bleached an entire Plin4-oil particle. In this case, fluorescence recovery should occur by exchange between free Plin4 12mer-A488 in solution and bleached Plin4 12mer-A488 molecules bound to the lipoparticle surface. As shown in Figure 1D, we detected no recovery within the time range of the measurement $(3 \mathrm{~min})$. In the second FRAP protocol, we bleached a limited area of the lipoparticle surface to follow fluorescence 
recovery of Plin4 12mer molecules by lateral diffusion. Again, we observed no recovery within 5 minutes after bleaching (Figure 1D). Thus, Plin4 12mer forms a very stable and immobile coat at the surface of triolein.

To gain further insight into the stability of the Plin4 $12 \mathrm{mer} /$ triolein particles, we visualized the emulsions over 7 days after the vortexing step. As shown in Figure $1 \mathrm{E}$, the emulsions at $\mathrm{t}=15$ $\min , 75 \mathrm{~min}, 4$ days and 7 days appeared very similar, showing numerous submicrometer particles and a few larger $(>1 \mu \mathrm{m})$ particles. Importantly, most if not all particles remained isolated during this long observation time, showing no obvious clustering or aggregation.

For comparison, we used a mutated form of Plin4 $4 \operatorname{mer}(4 T>S)$, in which several threonines in the hydrophobic face of the amphipathic region had been replaced by the more polar residue serine (Figure 1A). Plin4(4T>S) was purified from bacteria following the same procedure as for Plin4 4mer or Plin4 12mer. This mutant was inefficient at emulsifying olein (Figure 1C), in agreement with its inability to target LDs in HeLa cells (Figure 1B) (Copic et al., 2018). However, we could observe a few large triolein droplets formed by fluorescently-labeled Plin4(4T>S). The lipoparticles covered by Plin4(4T>S) clustered over time, suggesting that their coat was much less protective than that observed with Plin4 12mer (Figure 1E).

Analysis by DLS revealed an even more remarkable stability of Plin4 12mer-oil particles over time, as we detected no change in particle size distribution even 28 days after emulsification (Figure 1F). This puts Plin4 AH on par with natural emulsifiers used for technological purposes in food or pharmaceutical industry (McClements and Gumus, 2016). In contrast, the particles formed by Plin4(4T>S) were too heterogenous for analysis by DLS even at the first time-point (3h) after emulsion formation.

\section{Following Plin4-oil interaction in real time using microfluidics}

Large energies are required to disperse oil in order to form oil-protein emulsions in vitro; in our case, we provided this energy through vigorous vortexing. To further study the interaction of Plin4 $\mathrm{AH}$ with neutral lipids, we required a method where we could present the $\mathrm{AH}$ to the oil surface in a gentle manner and follow in real time the assembly of protein on the oil surface. We developed a microfluidics system, in which we used a glass chip with two channels joined 
by a T-junction. We introduced the water-based buffer into the main channel and pure triolein into the side channel and stabilized the buffer-triolein interface 50-100 $\mu \mathrm{m}$ below the Tjunction by closing the valve in the side-channel (Figure 2A). In this configuration, the buffertriolein interface is not disturbed by the flow in the main channel, whereas the solutes from the main channel are free to diffuse to the oil surface. In terms of diffusion and hydrodynamic characteristics, this system is similar to microfluidic cavities (Osterman et al., 2016; Vrhovec et al., 2011).

We introduced Alexa-488-labeled Plin4 12mer into the main channel, and we followed the change in fluorescent signal inside the side channel and on the triolein interface over time using a confocal microscope (Figure 2B and Supplementary movies). As the protein solution in the main channel reached the T-junction we could observe its diffusion into the side channel. After several seconds, we detected an increase in fluorescence on the oil interface, which stabilized in $\approx 3 \mathrm{~min}$ at a level 3-fold higher than the fluorescence of the solution (Figure $2 \mathrm{C}$ ). We then replaced the protein solution in the main channel with buffer to promote protein dissociation. However, the fluorescence at the interface remained constant, indicating a stable interaction between Plin4 12mer and oil. No enrichment of fluorescence on the oil interface was observed when we introduced buffer containing Alexa488 conjugated to free cysteine instead of Plin4 12mer (lower row in Figure 2B,C). These experiments confirm that Plin4 AH forms a very stable protein layer at the oil/water interface.

We compared the interaction Plin4 12mer with oil to that of the less hydrophobic Plin4(4T>S) mutant using the microfluidics system. The mutant assembled on the oil surface with no measurable difference in the kinetics of assembly or in the factor of enrichment compared to Plin4 12mer. However, the difference between Plin4 12mer and Plin4(4T>S) became obvious when the two proteins were used as unlabeled proteins in a 50:1 molar excess over labeled Plin4 12mer. We observed strong fluorescent signal on the oil interface in the presence of the Plin4(4T>S) mutant, but not in the presence of Plin4 12mer, indicating that the wild-type protein out-competed with Plin4(4T>S) for oil coating (Figure S1). 


\section{Comparison between the $\mathrm{AH}$ of Plin4 and other perilipins}

So far, we focused on the interaction between the AH of Plin4 and LDs as it represents a most striking example of an LD-binding $\mathrm{AH}$. We wanted to specifically compare the characteristics and LD-binding properties of Plin4 $\mathrm{AH}$ with the $\mathrm{AH}$ regions of the other human perilipins (Plin1, Plin2, Plin3), which have been shown to contribute to LD targeting (McManaman et al., 2003; Nakamura and Fujimoto, 2003; Bulankina et al., 2009; Rowe et al., 2016). The number of 11aa repeats that we could identify in each protein ranged from 5 for Plin5 to about 8 for Plin1/2/3, compared to the 87 repeats in Plin4. In addition, the repeats are more highly conserved in Plin4, and Plin4 AH is also striking for the absence of any deletions or insertions between the repeats (Copic et al., 2018) (Figure 3A). Comparison of the composition of the 11-aa regions showed that they were similar in character in Plin2/3/4, with low hydrophobicity due to a lack of large hydrophobic residues (Figure 3B). Plin1 $\mathrm{AH}$ is somewhat more hydrophobic and contains some aromatic residues. A more divergent character of this $\mathrm{AH}$ is consistent with the evolutionary divergence of Plin1 from the other perilipins (Granneman et al., 2017). The AH of Plin5 is shorter than in other perilipins and we did not consider it in further analysis.

We first expressed Plin AHs as GFP fusions in budding yeast and assessed their ability to target LDs. Budding yeast was used previously for expression of mammalian perilipins; full-length Plin1, Plin2 and Plin3, as well as their N-terminal halves, which include a region termed 'PAT domain' in addition to 11-aa repeats, targeted LDs in this system (Jacquier et al., 2013; Rowe et al., 2016; Copic et al., 2018). We expressed the AHs of Plin1, Plin2, and Plin3, and fragments of different lengths from the $\mathrm{AH}$ region of Plin4, containing 4, 6, or 12 33-aa repeats $(132,198$ and 396 aa, respectively). In the case of Plin3, we could not observe any expression of just the AH region (aa 113-205) fused to GFP, therefore we added some additional upstream sequence (aa87-205) (Bulankina et al., 2009). We expressed these constructs under three growth conditions that promote LD accumulation (Gao et al., 2017) : (i) wild-type cells grown to stationary phase; (ii) stationary phase cells lacking the most abundant yeast LD protein,

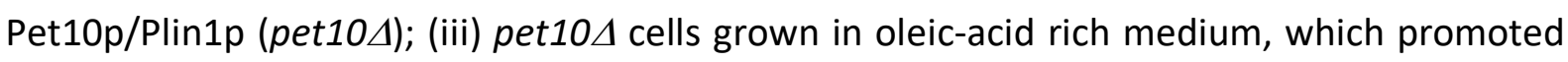
the formation of large LDs (pet10 + OA). In wild-type cells, Plin1 AH, but not Plin2 AH, Plin3 AH or Plin4 4mer, could be observed on LDs (Figure 3C). In contrast to Plin4 4mer, Plin4 6mer 
and Plin4 12mer localized to LDs, in line with our finding that increasing the AH length improves LD targeting (Copic et al., 2018). In agreement with the work of Gao et al., deletion of Pet10p/Plin1p improved LD targeting of our mammalian constructs, presumably because more LD surface was available (Kory et al., 2015). Targeting to LDs was further increased by the addition of oleic acid to stationary phase cells, which induced large LDs (Figure 3C). In addition, we observed some protein at the PM, in particular in the case of longer Plin4 AHs, consistent with observations from human cells and tissues (Scherer et al., 1998; Ruggieri et al., 2020). Based on these results, we conclude that the 11-aa repeat regions of Plin1, Plin2, Plin3, and Plin4 are all sufficient for targeting LDs. Comparison of different growth conditions (Figure 3D) allowed us to establish a ranking of LD affinities: extrapolating to its full length, Plin4 AH has the highest affinity for LDs, followed by Plin1 AH, and finally by Plin2 AH and Plin3 $\mathrm{AH}$. However, correcting for length differences reveals that per unit of $\mathrm{AH}$ length, Plin1 $\mathrm{AH}$ has a higher affinity for LDs than those of Plin2, Plin3 or Plin4. This is consistent with the higher hydrophobicity of Plin1 AH compared to other perilipin AHs; higher hydrophobicity has been shown to promote LD binding (Copic et al., 2018; Prévost et al., 2018).

Strikingly, we noticed a difference in the size of the large LDs that formed in pet10 $\Delta$ cells grown in oleic acid-rich medium, depending on the $\mathrm{AH}$ expressed (Figure $3 \mathrm{C}, \mathrm{E}$ ): LDs were significantly larger (2.5-fold difference in projected area, which would correspond to a 4-fold difference in volume) when covered with Plin1, Plin2 or Plin3 AH, compared to LDs covered with Plin4 12mer (Figure 3C,E). LDs with Plin4 6mer were also somewhat bigger than those covered with Plin4 12mer. LDs with Plin4 4mer were more variable in size and appearance, preventing the use of the same quantification protocol.

We conclude that AHs from all four perilipins (Plin1-4) can target LDs and that their affinity for LDs correlates with their length and hydrophobicity. However, the AH of Plin4 could reduce the size of LDs more strongly than the AHs of other perilipins.

\section{Stability of binding of perilipin AHs to LDs in model cellular systems}

We used FRAP to assess the stability of AH binding to LDs using the yeast model system. Plin1 AH-GFP could readily exchange between LD surface and the cytosol in cells grown for $24 \mathrm{~h}$ in oleic acid medium, as reflected by a recovery half-life on the order of a few seconds (Figure 
4A). The exchange of Plin3 AH-GFP was about two times faster than Plin1 AH-GFP, consistent with a lower amount of binding to LDs at steady state and with results obtained in mammalian cells with N-terminal halves of Plin1 and Plin3 (Ajjaji et al., 2019). In striking contrast, Plin4 12mer-GFP displayed almost no recovery on LDs over a period of more than 5 min (Figure 4A). We obtained similar measurements in cells grown for a shorter time, to early stationary phase, in standard growth medium (Figure 4B). However, these LDs were much smaller and more mobile, leading to a large variability in the fluorescence measurement. We also performed FRAP of Plin4 AH-GFP constructs bound to the PM in cells in exponential phase, to ensure that the poor recovery times on LDs were not due to protein aggregation in aged cells. The results for Plin4 12mer-GFP on the PM were qualitatively similar to the results on LDs, showing slow and incomplete recovery of fluorescent signal after photobleaching. The recovery was faster and more complete for shorter Plin4 $\mathrm{AH}$ constructs (8mer and 6mer), confirming that the length of the Plin4 AH contributes to its binding to lipid surfaces (PM or LD) in the yeast model system (Figure 4C). However, even for the shortest Plin4 $\mathrm{AH}$ construct for which we consistently observed targeting to yeast membranes, the recovery after photobleaching was still an order of magnitude slower than for Plin1 $\mathrm{AH}$ or Plin3 $\mathrm{AH}$, suggesting that length of Plin4 $\mathrm{AH}$ is not the only parameter determining its stable binding to LDs.

Next, we moved to Drosophila Schneider 2 (S2) cells, which have been used to decipher mechanisms of LD homeostasis (Guo et al., 2008; Krahmer et al., 2011). We previously demonstrated that expression of Plin4 12mer in S2 cells rescued the increase in size of LDs following the depletion of phosphatidylcholine (PC) (Copic et al., 2018). In these cells, the FRAP recovery curves of Plin4 12mer-GFP on LDs were variable, with a half-time covering a full range between $1 \mathrm{~s}$ to $>100 \mathrm{~s}$ (Figure S2). However, this variability was cell-dependent: within the same cell, the FRAP curves from different LDs were similar, suggesting that a cell-to-cell dependent variable was at play. PC depletion upon CCT $\alpha$ knocked-down had a small effect, but this was not the main driver of cell-to-cell variability. The level of protein expression was also not very predictive of recovery rate. In contrast, we observed a correlation between the rate of FRAP recovery and the intensity of the Plin4 fluorescent signal on LDs. This observation suggests that Plin4 AH density at the LD surface influences its dynamics, a feature reminiscent of protein coat. At low membrane coverage level, coat subunits diffuse and exchange quickly; 
at high membrane coverage level, their polymerization by side-side interaction prevents lateral mobility and fast turnover (Saleem et al., 2015; Sorre et al., 2012).

\section{Comparison of proteolipid droplets formed with Plin4 AH or Plin3 $\mathrm{AH}$}

To study in more detail the difference between Plin4 and other perilipin AHs binding to LDs, we used our in vitro assays to compare the behavior of purified Plin4 $\mathrm{AH}$ fragments with that of Plin3 $\mathrm{AH}$. We chose Plin3 $\mathrm{AH}$ because it displayed a similar steady-state distribution in yeast as the slightly longer Plin4 4mer, however, it showed a rapid exchange between LDs and the cytosol and it did not decrease LD size in oleic acid media. Mixing purified Plin3 AH with oil resulted in a highly turbid suspension, similar to the suspensions obtained with Plin4 4mer or Plin4 12mer (Figure 5A and Figure S3). By DLS, Plin4 4mer-oil droplets behaved like Plin4 12mer oil droplets (see Figure 1G) (Copic et al., 2018), displaying a particle size profile with a single peak that did not change over 14 days (Figure 5B, left panel). In contrast, the droplets produced by Plin3 $\mathrm{AH}$ were more heterogenous already $3 \mathrm{~h}$ after droplet formation. Thereafter, we observed a spreading of the peaks until the samples became too complex for DLS analysis (14 days after formation Figure 5B, right panel). Such complexity is generally due to the presence of particles of variable sizes, suggesting that Plin3 $\mathrm{AH}$-oil particles were undergoing fusion due to less stable coating by Plin3 $\mathrm{AH}$.

Centrifugation of $\mathrm{AH}$-oil suspensions on sucrose gradients revealed a smaller fraction of total Plin3 AH protein associated with the oil fraction (top of the gradients) than Plin4 4mer or Plin4 12 mer, even though we used the same protein to oil mass ratio (Figure $5 C, D$ ). This could be either because less Plin3 $\mathrm{AH}$ was bound to the oil droplets or because Plin3 $\mathrm{AH}$ bound to oil less strongly and dissociated during centrifugation. To distinguish between these possibilities, we performed competition experiments in which we first formed protein-oil droplets by mixing oil with a high concentration of unlabeled purified AH constructs (Plin4 12mer, Plin4 4mer or Plin3 $\mathrm{AH}$ ). Then, we gently added Alexa488-labeled Plin4 12mer at an excess mass ratio of 20:1 compared to unlabeled protein, and we monitored the fluorescence of the suspensions over time using confocal microscopy (Figure 5E). We observed no incorporation of fluorescent Plin4 12mer into the preformed Plin4 12mer-oil particles over a period of $24 \mathrm{~h}$, unless we vortexed the suspension (Figure 5E,F; top panel). This result is consistent with the lack of Plin4 12mer dissociation from oil, as determined by FRAP or by microfluidics (Figure 1D 
and Figure 2B). In contrast, we observed some incorporation of fluorescent Plin4 12 mer into Plin4 4mer-oil particles after 3 or 24 hours of incubation, suggesting that Plin4 4 mer was less stably bound to oil than the 3-times longer Plin4 12mer (Figure 5E,F; middle panel). Strikingly, when we pre-formed AH-oil particles using Plin3 AH, Plin4 12mer much more quickly incorporated into these particles, reaching close to maximal particle fluorescence already 10 min after Plin4 12mer addition (Figure 5E,F; bottom panel). We also observed clustering of Plin3 AH-formed oil particles, especially after 24 hours of incubation. Together, these experiments suggest that like Plin4 $\mathrm{AH}$, Plin3 $\mathrm{AH}$ can function as an oil emulsifier. However, Plin3 binding to oil is much less stable and the Plin3-oil particles cluster and fuse over time. The stability of Plin4-oil particles is promoted by the length of the Plin4 AH. However, Plin4 4mer, which is less than 1.5-times longer than Plin3 AH, also produced much more stable oil droplets. Thus, the difference in the stability of Plin4-oil and Plin3-oil droplets is not only due to the length of Plin4 $\mathrm{AH}$, but also to differences in the $\mathrm{AH}$ sequences.

\section{The nature and distribution of aa in the polar face of Plin4 AH is critical for LD targeting}

The Plin4 AH sequence displays a remarkable repetitiveness (Figure $3 \mathrm{~A})$. Positions of polar and charged residues are extremely conserved among the repeats (Figure 6A). Furthermore, the sequence shows a strong preference for lysine over arginine (22-fold) and for aspartic over glutamic acid (18-fold) (Figure 3B). These considerations prompted us to construct mutants of Plin4 4mer in which we introduced in every 33-aa repeat modest mutations (e.g. $N>Q, D>E$, or $\mathrm{K}>\mathrm{R}$ ) that should not modify the folding and overall physical chemistry of the helix, including its charge and hydrophobic moment.

We first focused on the two conserved amide residues: an asparagine present in 25 out of 29 repeats of human Plin4, and a glutamine seven residues apart and conserved in all repeats (Figure $6 A$ ). The $N[x]_{6} Q$ sequence was replaced by $N[x]_{6} N(N N), Q[x]_{6} Q(Q Q)$ or $Q[x]_{6} N(Q N)$. Strikingly, these three mutations almost eliminated the targeting of Plin4 4mer to LDs in HeLa cells (Figure 6C). Next, we considered the charged residues. Replacing all aspartates with glutamates $(2 \mathrm{D}>\mathrm{E})$ led to a small decrease in $\mathrm{AH}$ targeting to LDs in HeLa cells, whereas replacing the lysine residues with arginine $(3 \mathrm{~K}>\mathrm{R})$ almost abolished $\mathrm{AH}$ targeting to LDs (Figure $6 \mathrm{~B}, \mathrm{C})$. These results suggested that a precise interaction between charged and/or polar residues could be important for LD binding. 
We also noted an unusual distribution of charged residues throughout the Plin4 $\mathrm{AH}$ sequence, with positive ones always lying on one side of the helix close to the apolar/polar interface (Figure 6D). To test whether charged residues in Plin4 $\mathrm{AH}$ could mediate interhelical interaction at the LD surface, we prepared a mutant of Plin4 12mer in which we reorganized the distribution of charges in the polar face of all 33-mer repeats without changing the overall composition (Figure 6D). This more symmetric Plin4 12mer AH mutant, termed charge-swap (csw), was similar to a 4mer mutant that we tested previously and which did not localize to LDs when expressed in HeLa cells (Copic et al., 2018). We observed some localization of the longer csw 12 mer-GFP mutant to LDs in HeLa cells, which was significantly reduced compared to Plin4 12mer-GFP (Figure 6E). When we compared the localization of these two constructs in the yeast model, we observed a difference in their distribution between LDs and the PM, with csw 12mer showing a lower ratio of LD-to-PM signal compared to Plin4 12mer (Figure 6F). This preference for the PM is consistent with the distribution of positive charges in csw $12 \mathrm{mer}$, which is optimal for mediating electrostatic interactions with the negative surface of the PM. Remarkably, when we performed FRAP of Plin4 12mer-GFP and csw 12mer-GFP bound to the PM in exponentially-growing cells, we observed a faster recovery of the csw construct compared to WT (Figure 6G). The observation that even though the csw mutant partitioned more strongly to the PM, its binding to the PM was more dynamic than in the case of Plin4 12mer, was suggestive of two separate kinetic steps: initial binding of individual helices followed by their assembly into a stable lattice. We could not perform a similar comparison on LDs due to their mobility. However, we noted a small shift towards bigger LDs in LDs surrounded by csw 12mer, compared to Plin4 12mer (Figure S4). Together, these results suggest that reorganization of charged residues in the Plin4 AH decreased the stability of helices bound to the LD surface.

We purified csw $12 \mathrm{mer}$ following the same purification procedure as for Plin4 AHs, and we tested the interaction of this mutant with oil. Like Plin4 $\mathrm{AH}$, csw 12 mer could produce oil droplets in our vortexing assay (Figure 7A). However, when we added Alexa488-labeled Plin4 12 mer to preformed csw-12mer-oil droplets, we observed a significant incorporation of Alexa488 fluorescence into the droplets (Figure 7B,C), in contrast to the lack of exchange observed between non-fluorescent and fluorescent Plin4 12mer (Figure 5E,F). We conclude that the 
particular aa distribution in the polar face of the Plin4 AH enables the formation of a highly stable structure on a neutral lipid surface (Figure 7D).

\section{Length and precise organization of Plin4 $\mathrm{AH}$ were selected during mammalian evolution}

Plin4 is a mammalian specific protein (Granneman et al., 2017). We asked how the sequence of Plin4 $\mathrm{AH}$ has evolved during mammalian evolution. Comparison of 16 mammalian Plin4 orthologs from different mammalian lineages (Bininda-Emonds et al., 2007) showed variability in the length of the $\mathrm{AH}$, i.e. the number of 33-aa repeats (Table S1 and Figure 8). Plin4 from the tree shrew Tupaia chinesis displayed the lowest number of repeats (22), whereas the highest number of repeats (58) was present in the bat Myotis davidii. The latter protein would therefore contain an $\mathrm{AH}$ of close to 2000 aa! In most orthologs, all repeats were consecutive and we found only a few insertions or deletions between individual repeats (Table S1). Furthermore, alignment of the 33-aa repeats shows a high conservation within the same Plin4 ortholog (Figure 8). The conservation of repeats is the lowest in platypus (O. anatinus), the most basally branching of the compared mammalian species, whereas the highest conservation is found in the bat $M$. brandtii. Charged and polar residues are highly conserved in the majority of species. Interestingly, when a mutation in a conserved residue appears in one species, it is usually propagated within the repeats. Note in particular $\mathrm{N}$ in position 21 (Figure 8), which is mutated to $G$ in some species of the Laurasiatheria lineage (BinindaEmonds et al., 2007).

These results suggest an increased rate of evolution of the Plin4 $\mathrm{AH}$ sequence in mammals and at the same time a strong functional constraint on its repetitive structure. Altogether, our results show that the precise organization of Plin4 $\mathrm{AH}$, in addition to its length, is important for Plin4 function in mammalian cells. 


\section{Discussion}

Perilipins are among the most abundant proteins in the LD proteomes (Brasaemle et al., 2004; Sztalryd and Brasaemle, 2017). Whereas their importance for LD metabolism has been known for a long time, notably for the recruitment of lipases and their inhibitors, this does not explain their abundance on the LD surface. The role of Plin4, by far the largest of perilipins, has been particularly puzzling. Plin4 is a mammalian-specific protein and is highly expressed in adipocytes (Wolins et al., 2003). Its deletion in a mouse model has so far not revealed any strong phenotypes (Chen et al., 2013). However, the striking features of Plin4 AH in terms of its length, repetitiveness and particular aa composition, and the variability in Plin4 $\mathrm{AH}$ sequence in mammals, suggest a strong selection for a specific function. Our experiments show that the interaction of Plin4 AH with LDs in vitro and in cellular model systems is remarkably stable. The slow lateral diffusion and the very slow dissociation of Plin4 $\mathrm{AH}$ molecules at the LD surface as assessed by FRAP, by microfluidics, or by exchange assays, are reminiscent of the behavior of vesicular coat components that polymerize on a membrane surface via lateral interactions (Saleem et al., 2015; Sorre et al., 2012).

We previously showed that the extreme length and the low hydrophobicity of Plin4 $\mathrm{AH}$ contributed to the specificity of its LD targeting (Copic et al., 2018). Large residues such as F or $\mathrm{W}$ are rare or absent in the Plin4 $\mathrm{AH}$ sequences, whereas three small hydrophobic residues, $\mathrm{V}, \mathrm{T}$ and $\mathrm{A}$, are extremely abundant (Figure 3B and Figure 8). Mutations that slightly increased hydrophobicity $(T>V)$ made Plin4 promiscuous for other organelles besides LDs, whereas mutations that slightly decreased hydrophobicity $(T>S)$ made Plin4 cytosolic. We now show that the Plin4(4T>S) mutant is also unable to emulsify oil in vitro although it binds to an exposed oil surface in microfluidics experiments. Thus, the hydrophobicity of Plin4 AH appears at the threshold of promoting LD binding. Our experiments in the yeast model (Figure 3C-E) show that this is also the case for other perilipin AHs, consistent with their overall similar chemistries. A slight exception is the AH of Plin1, which contains some aromatic residues and partitions to LDs better than other AHs (Rowe et al., 2016; Ajjaji et al., 2019). Overall, the hydrophobicity of perilipin AHs appears at best modest and generally extremely low. As such, these proteins challenge a recent model for AH-LD interaction in which the main driving force is the intercalation of bulky hydrophobic residues within lipid packing defects at the LD surface 
(Prévost et al., 2018). This model derives from binding experiments and cellular observations performed with AHs that are much smaller than those of perilipins and bind not only to LDs but also other organelles (e.g. ALPS motif, CCT $\alpha$, Arf1). As such, this model does not account for the particular chemistry of Plin AHs, which are the most abundant and specific AHs of LDs.

Surprisingly, the polar face of Plin4 AH makes a very large contribution to LD targeting. The sequence conservation in this face, including a strong preference for K over R and D over $E$, suggests that polar and charged residues play an important role. All mutations that we tested, including $N>Q, Q>N, K>R$ and $D>E$, decreased Plin4 $A H$ LD targeting, with the first three mutations causing an almost complete dissociation. Furthermore, merely changing the distribution of these residues in the polar face led to a reduction in LD targeting and in stability of binding to triolein droplets in vitro. We thus propose that binding of Plin4 $\mathrm{AH}$ is controlled by the numerous electrostatic/hydrogen interactions that its polar side chains can engage in along its gigantic length. These features speak in favor of a 'coat' model: numerous Plin4 molecules held together by side-side interactions would form a network at the LD surface. A prediction of this model is that Plin4 should be immobilized at the LD surface and, thereby, should exhibit very limited dynamics. This was confirmed by our experiments, which revealed a drastic difference between Plin4 $\mathrm{AH}$ and other perilipin AHs in cells and in vitro.

The coat model of Plin4 is reminiscent of the interactions that apolipoproteins engage in to form secreted lipoprotein particles (Phillips, 2013). ApoA1, for which most structural information is available, forms a ring around the lipid core in low density lipoprotein particles, stabilizing itself via interactions between charged residues from two adjacent molecules (Bibow et al., 2017; Pourmousa et al., 2018; Melchior et al., 2017). 26 intermolecular saltbridges connect two antiparallel rings of ApoA1, which is about 200 aa long. The abundance of positively (3) and negatively (2) charged residues in each 33-aa repeat of Plin4 is compatible with the formation of a similar large network of intra or intermolecular interactions. Interestingly, this model does not impose a strict geometry for the protein network. The large number of glycine residues in Plin4 AH (5 G per 33-aa repeat) could enable the formation of various turns, resulting in a spaghetti-like layer rather than a geometrically well-defined assembly. 
The observation that perilipins decorate different LDs in the same cell type supports our model of a higher-order perilipin organization (Hsieh et al., 2012; Wolins et al., 2005). The network of electrostatic interactions between perilipin AHs should be strongly dependent on their exact sequences, making the formation of hybrid coats with different perilipins less likely than homogenous perilipin coats. However, what drives the sequential coating of LDs by different perilipins remains mysterious. In addition to their repetitive $\mathrm{AH}$ regions, other segments of Plin1, Plin2 and Plin3 have been implicated in binding to LDs, in particular the Cterminal 4-helix bundle (Subramanian et al., 2004; Mirheydari et al., 2016; Ajjaji et al., 2019), but this has not been the case for Plin4 (Copic et al., 2018). In contrast to the behavior of their AHs, the association of full-length Plin1 and Plin2 with LDs can be very stable (Targett-Adams et al., 2003; Soni et al., 2009; Pataki et al., 2018; Ajjaji et al., 2019), and the COPI machinery has been implicated in the recruitment of Plin2 to LDs by an unknown mechanism (Nakamura et al., 2004; Soni et al., 2009).

Whereas vesicular coats uniformly cover the surface of vesicles, this is unlikely to be the case for perilipin coats. Non-uniform distribution of Plin1 on LD surface has been observed in cultured adipocytes (Blanchette-Mackie et al., 1995; Hansen et al., 2017); this can be explained by a coating model, where patches of polymerized perilipin coat might coexist with LD regions decorated by other proteins. More generally, deciphering the molecular arrangements of Plin4 molecules or other perilipins on LDs is a considerable challenge for the future. In the case of apolipoproteins, a consensual model for their organization is just starting to emerge despite decades of intense investigations on these proteins.

The sequence of Plin4 is particularly striking in light of a recent study that identified an increased number of homologous repeats (10 additional repeats) in the Plin4 AH encoding region of individuals from a single family with a rare muscular degeneration (Ruggieri et al., 2020). The study proposes that a higher number of repeats in Plin4 leads to protein aggregation in muscle cells, suggesting that the sequence of Plin4 represents a risk for the organism. It is then even more surprising that a protein with such a long and repetitive $\mathrm{AH}$ has arisen during the mammalian evolution. While our results suggest that such a configuration represents a means of guarding the stability of LDs, the precise advantages for mammalian cellular metabolic pathways remain to be discovered. 


\section{Materials and Methods}

\section{Sequence analysis}

The 11-aa repeats of perilipins and 33-aa repeats of Plin4 orthologues were identified using HHrepID tool from the MPI Bioinformatics Toolkit server (Biegert and Söding, 2008; Zimmermann et al., 2018). The amphipathic character of these sequences was analysed using HeliQuest (Gautier et al., 2008). Helical wheels were plotted as complete 3-11 helices; the presentation of helices was chosen such as to maximise their hydrophobic moment, as calculated by Heliquest, and inclusion of identified 11-aa repeats, excluding helix-breaking proline (Pace and Scholtz, 1998) from the middle of the helices. The amino acid conservation of the 33-aa repeats of Plin4 was represented using Weblogo (Crooks et al., 2004).

\section{Plasmid DNA construction}

All plasmids used in this study are listed in Table S2. DNAs encoding AHs of human Plin1, Plin2 and Plin3 were PCR-amplified from the corresponding cDNAs that had been cloned into pGREG576 plasmids (gift from R. Schneiter, U. of Fribourg) (Jacquier et al., 2013). DNA for Plin4 6mer and Plin4 8mer was amplified from plasmid pCLG26, and DNA for Plin4 4mer mutant 4T>S was amplified from plasmids pSB49 (Copic et al., 2018). Plin4 4mer mutants $(2 \mathrm{D}>\mathrm{E}, 3 \mathrm{~K}>3, \mathrm{NN}, \mathrm{QN}$ and $\mathrm{QQ})$, and Plin4 12mer mutant csw 12 mer were constructed using synthetic double-stranded DNA fragments (Table S3). All 4mer mutants were exact $4 \times$ repeats of a 33-aa sequence, based on the parental sequence of human Plin4 fragment aa246-278. The protein sequence for csw 12 mer was designed by manually adjusting 33-aa helical wheels of the parental Plin4 12mer sequence using HeliQuest to increase the symmetry of charged residue distribution in the polar side of the helix while minimizing changes in the hydrophobic moment. DNA sequences were optimized for synthesis using the algorithm on the Eurofins website (https://www.eurofinsgenomics.eu). Table S2 also lists all protein sequences used in this study.

For expression of proteins in E. coli, PCR-amplified DNA fragments were inserted into pET21b (Novagen) without adding a tag using Nhel and Xhol restriction sites, which were introduced by PCR. For expression of Plin3 $\mathrm{AH}$, an additional sequence 'MASC' was introduced upstream of the AH. For expression of GFP fusion proteins in S. cerevisiae, PCR-amplified DNA fragments were inserted into pRS416-derived (URA3 and AmpR markers) CEN plasmid pRHT140 
containing ADH1 promoter and GFP for C-terminal tagging (gift from S. Leon, IJM). For expression of mCherry fusion proteins, GFP-encoding fragment in this vector was replaced with mCherry using $\mathrm{Kpnl}$ and $\mathrm{BamHI}$ restriction sites to generate plasmid pMGA4. All AH DNA fragments were cloned into these plasmids using $\mathrm{Nhel}$ and $\mathrm{BamHI}$ restriction sites that were introduced by PCR. The sequence of the multiple cloning site introduces a linker peptide in the resulting fusion protein between the AH and GFP, 'PLDPPGLQEF', and linker peptide 'VKDPDIKLID' between the $\mathrm{AH}$ and mCherry. Plasmids for expression of mCherry fusion proteins in HeLa cells were constructed by subcloning synthetic genes for Plin4 mutants into pmCherry-N1 (Invitrogen) using BamHI and Xhol restriction sites. All plasmids were verified by sequencing.

\section{Protein purification}

All proteins were purified from E. coli without a tag. Plin4 12mer and Plin4 4mer were purified as previously described (Copic et al., 2018). Plin3 AH (aa103 to 205), Plin4 4T>S and csw $12 \mathrm{mer}$ were purified following a similar protocol, with some modifications in the case of Plin $3 \mathrm{AH}$, as outlined below. E. coli cells BL21DE3 transformed with expression plasmids were grown to O.D. $\approx 0.6$ at $37^{\circ} \mathrm{C}$ from a liquid preculture and induced with $1 \mathrm{mM}$ IPTG for $1 \mathrm{~h}$ at $37^{\circ} \mathrm{C}$. Cells from 0.25 I cultures were collected by centrifugation and frozen. The bacterial pellets were thawed in lysis buffer (50 mM Tris- $\mathrm{HCl}$ pH 7.5, $150 \mathrm{mM} \mathrm{NaCl}, 1 \mathrm{mM}$ DTT, supplemented with $0.1 \mathrm{mM}$ PMSF, and Complete protease inhibitor cocktail (Roche)). Cells were broken by sonication. The lysate was centrifuged at $100,000 \times \mathrm{g}$ for $30 \mathrm{~min}$ at $4^{\circ} \mathrm{C}$ in a $70.1 \mathrm{Ti}$ Rotor $(40,000$ rpm; Beckman). The supernatant in centrifuge tubes was immersed in boiling water $\left(95^{\circ} \mathrm{C}\right)$ for $30 \mathrm{~min}$. The resulting cloudy suspension was centrifuged at $100,000 \times \mathrm{g}$ for $15 \mathrm{~min}$ at $4^{\circ} \mathrm{C}$ to remove precipitated material. The supernatant was dialyzed against $20 \mathrm{mM} \mathrm{Tris-} \mathrm{HCl} \mathrm{pH} 7.5$, $10 \mathrm{mM} \mathrm{NaCl}, 1 \mathrm{mM}$ DTT ( $2 \times 30$ min using $1 \mathrm{I}$ of buffer) at $4{ }^{\circ} \mathrm{C}$ using Spectra/Por membranes with a cut-off of $6000 \mathrm{Da}$ (Spectrum labs) and then centrifuged again at 100,000 $\times \mathrm{g}$ for $30 \mathrm{~min}$ at $4{ }^{\circ} \mathrm{C}$. Plin4 $4 \mathrm{~T}>\mathrm{S}$ and $\operatorname{csw} 12 \mathrm{mer}$ were then further purified in a single step by cation exchange chromatography on a $20 \mathrm{ml}$ Hiprep S HP column (GE Healthcare), as described for Plin4 AHs (Copic et al., 2018). In contrast to Plin4 AH, Plin3 AH has a net negative charge at neutral $\mathrm{pH}(\mathrm{pl}=4.65)$. Therefore, Plin3 $\mathrm{AH}$ was purified by anion exchange chromatography on a $20 \mathrm{ml}$ Hiprep Q HP column (GE Healthcare). It was eluted with a salt gradient from 10 $\mathrm{mM}$ to $400 \mathrm{mM} \mathrm{NaCl}$ (3 column volumes) in $20 \mathrm{mM}$ Tris- $\mathrm{HCl} \mathrm{pH} 7.5,1 \mathrm{mM}$ DTT at a flow rate 
of $2 \mathrm{ml} / \mathrm{min}$ using an Akta purifier system (GE Healthcare), eluting at approximately $100 \mathrm{mM}$ $\mathrm{NaCl}$. After analysis of the chromatography fractions by protein electrophoresis, the protein pools were divided in small aliquots and stored at $-80^{\circ} \mathrm{C}$.

\section{Protein electrophoresis and determination of protein concentration}

Standard Glycine SDS-PAGE was used for the analysis of Plin4 12mer and csw 12mer (Mw 40 kDa) using homemade 13\% acrylamide-bisacrylamide gels. Tricine SDS-PAGE (Schägger and Jagow, 1987) was used for proteins with lower molecular weight, i.e. Plin4 4mer, Plin4 4T>S, or Plin3 AH (9-15 kDa). For that we either used TruPAGE commercial gels (Sigma) and homemade Tris-MOPS buffer (60 mM Trizma, 30 mM 4-Morpholinepropanesulfonic acid (MOPS), $0.1 \% \mathrm{w} / \mathrm{v}$ SDS), or, for better resolution, homemade $16.5 \%$ acrylamidepolyacrylamide (29:1) gels run with tricine buffer (100 mM Tris-HCl pH 8-8.5, $0.1 \mathrm{M}$ Tricine, $0.1 \%$ SDS) in the cathode and $200 \mathrm{mM}$ Tris $\mathrm{HCl} \mathrm{pH} 8.9$ in the anode chamber. Gels were rinsed in $7.5 \%$ acetic acid, stained with SyprOrange (Life Technologies) and visualized with a MP imaging system (Bio-Rad) using the Alexa 488 settings. Because all perilipin AH purified construct lack aromatic residues, preventing protein quantification by UV spectroscopy or by Coomassie Blue, protein concentration was routinely determined by densitometry of SyproOrange stained gels against a calibration curve with protein standards (Sigma) using ImageJ. Quantification by gel electrophoresis was verified by Ellman's reaction method as previously described (Copic et al., 2018).

\section{Protein labelling with fluorescent probes}

Purified AHs were covalently labeled via cysteines using Alexa C5 maleimide probes (either 488 or 568; Thermofisher). Plin4 12mer, Plin4 $4 \mathrm{mer}$ and Plin4 $4 \mathrm{~T}>\mathrm{S}$ and csw 12mer were labeled on endogenous cysteines present in their AHs; they all contain 4 cysteines in total. Plin3 AH is devoid of cysteines, therefore a single cysteine was introduced at its $\mathrm{N}$-termus. To remove DTT, $1 \mathrm{ml}$ of protein solution at concentration $0.7 \mathrm{mg} / \mathrm{ml}(18 \mu \mathrm{M}$ of Plin4 $12 \mathrm{mer}$ and csw $12 \mathrm{mer}, 50 \mu \mathrm{M}$ of Plin4 4mer and Plin4 4T>S) was exchanged into labelling buffer ( $20 \mathrm{mM}$ Tris- $\mathrm{HCl}$ pH 7.5, $100 \mathrm{mM} \mathrm{NaCl}$ ) using size exclusion NAP10 columns (GE Healthcare). Proteincontaining fractions were identified by protein electrophoresis and pooled. Protein solutions were incubated for 5 minutes at $4{ }^{\circ} \mathrm{C}$ with Alexa $\mathrm{C} 5$ maleimide probes at an equimolar ratio to their total number of cysteines ( $1 \mathrm{ml}$ reaction volume). The reactions were stopped by the 
addition of DTT to $10 \mathrm{mM}$ final concentration and loaded on NAP10 columns to separate the labeled protein from the excess of fluorescent probe. Fractions were analysed by protein electrophoresis. Fluorescence in the gel was directly visualized on ChemiDoc MP imaging system (Bio-Rad) either with Alexa 488 or SyproRuby (for Alexa 568) settings. Fractions with labeled protein were pooled, aliquoted and stored at $-80^{\circ} \mathrm{C}$. The same protocol was used for labelling of free cysteine, but without NAP10 purification steps.

We used FRAP assays on protein-oil emulsions (see below) with different ratios of labeled to unlabeled proteins to verify that the fluorescent label did not change the behaviour of the protein. This was not the case for labeled Plin3 $\mathrm{AH}$, thus we only used this protein in unlabeled form in our biochemical assays.

\section{Preparation of protein-oil emulsions}

Proteins were diluted to $0.5 \mathrm{mg} / \mathrm{ml}$ in freshly degassed HKM buffer (50 mM Hepes-KOH pH 7.2, $120 \mathrm{mM} \mathrm{K}$-acetate, $1 \mathrm{mM} \mathrm{MgCl}$ ) supplemented after degassing with $1 \mathrm{mM} \mathrm{DTT.} 190 \mu \mathrm{l}$ of each solution were pipetted into a $600 \mu$ l glass tube, and a $10 \mu \mathrm{l}$ drop of triolein (>99\% purify, T7140 Sigma) was added to the top. In some cases, emulsions were prepared to have a final volume of $100 \mu \mathrm{l}$ and the drop of triolein was $5 \mu \mathrm{l}$. They were vortexed manually at a fixed angle of $\sim 30^{\circ}$ for three cycles of $30 \mathrm{~s}$ on $30 \mathrm{~s}$ off at $25^{\circ} \mathrm{C}$ under argon atmosphere. Images of resulting emulsions were taken with a compact camera. For analysis by fluorescent microscopy, emulsions were prepared using a mixture of fluorescent and unlabeled protein at a mass ratio $1: 20$.

\section{Dynamic Light Scattering (DLS)}

Measurements of the mean hydrodynamic radius of the Plin4-oil droplets by dynamic light scattering were performed on a sample taken from the middle of the tube, avoiding any unreacted oil that remained at the top of the emulsion, at least $3 \mathrm{~h}$ after vortexing to prevent the interference of gas bubbles with the measurement. Subsequent samples at later time points were removed in the same manner without any additional mixing. Emulsion samples were diluted 100-fold in freshly degassed HKM buffer with 1mM DTT. Measurements were performed on a Zetasizer Nano ZS machine (Malvern) at $25^{\circ} \mathrm{C}$, and data were processed using the CONTIN method. 


\section{Observation of protein-oil interaction using microfluidics}

Microfluidic experiments were performed in a glass microfluidic chip with a T-junction geometry purchased from Dolomite (part \# 3000086 and 3000024). All channels had a rounded cross-section with a $100 \mu \mathrm{m}$ height and a $110 \mu \mathrm{m}$ width. Prior to the measurements, the channel walls were wetted with $100 \mu$ l of freshly degassed HKM buffer supplemented with $1 \mathrm{mM}$ DTT. The flows were driven and precisely controlled using a piezoelectric pressure control system (OB1 MK3, Elveflow), with typically applied pressures below 300 mbar. After wetting, the main and side inlet channels were filled with buffer and triolein (Sigma), respectively. Injection of oil in the side inlet channel was gently stopped with a manual valve (MV201, LabSmith) before the oil reached the junction (when the meniscus was approximately $400 \mu \mathrm{m}$ from the junction). In this way, the flow in the main inlet channel could be stopped without significantly affecting the meniscus of oil, and the inlet vial with buffer could be exchanged by a vial with fluorescent protein solution $(0.1 \mathrm{mg} / \mathrm{ml}$ in HKM buffer, mixed at a ratio $10: 1$ for unlabeled vs Alexa488-labeled protein). The sample volume in the vial was approximately $400 \mu \mathrm{l}$. The flow in the main inlet channel was then resumed and the diffusion of the protein from the main inlet channel into the side channel and its adsorption onto oil meniscus was monitored by time-lapse confocal microscopy for up to $30 \mathrm{~min}$ at a rate of 1 frame every 3 seconds (ECLIPSE TE2000-E, Plan Fluor 40x objective, EZC1 software, Nikon). Finally, the flow in the main inlet channel was stopped again, the inlet vial exchanged by a vial with buffer, and the main inlet channel was rinsed while monitoring the diffusion of the protein from the side channel. For competition experiments between Plin4 12mer and 4T>S mutant, proteins were mixed at a mass ratio 50:1 for unlabeled to labeled protein, Plin4 12mer: Plin4 12mer-Alex488 or 4T>S: Plin4 12mer-Alex488, with a total protein concentration of $0.1 \mathrm{mg} / \mathrm{ml}$. All experiments were conducted at room temperature. Between experiments, glass chips were regenerated by copious washing with $3 \%$ SDS at $50^{\circ} \mathrm{C}$, followed by distilled water, $3 \%$ TFD4 at $50^{\circ} \mathrm{C}$, distilled water and finally dried by air. Image analysis was performed with ImageJ/Fiji (Schindelin et al., 2012) and Matlab.

\section{Separation of Plin4-oil emulsion on sucrose gradients}

Emulsions were prepared as specified in a final volume of $300 \mu$ including $15 \mu \mathrm{l}$ of triolein and $0.5 \mathrm{mg} / \mathrm{ml}$ of protein. Next, $240 \mu \mathrm{l}$ of $60 \% \mathrm{w} / \mathrm{v}$ solution of sucrose in HKM buffer with $1 \mathrm{mM}$ DTT was mixed with $240 \mu \mathrm{l}$ of emulsion, avoiding any oil. $450 \mu \mathrm{l}$ of this suspension was loaded 
on the bottom of a centrifuge tube and overlaid with a step sucrose gradient consisting of 300 $\mu \mathrm{l}$ 20\%, $300 \mu \mathrm{l} 10 \%$ and $100 \mu \mathrm{l}$ 0\% sucrose in HKM buffer with $1 \mathrm{mM}$ DTT. The samples were centrifuged at 50,000 rpm (214,000 $\times \mathrm{g})$ in a Beckman swing-out rotor (TLS 55) for $80 \mathrm{~min}$ at $8^{\circ} \mathrm{C}$. Four fractions were carefully collected from the bottom with a Hamilton syringe, having the following volumes: $450 \mu \mathrm{l}, 300 \mu \mathrm{l}, 300 \mu \mathrm{l}$, and $100 \mu \mathrm{l}$, respectively. Equal volumes of all fractions were analysed by protein electrophoresis.

\section{Yeast growth and media}

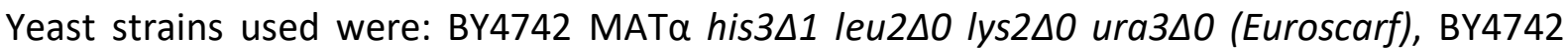
ERG6-mRFP::KanMX6 (Jackson lab collection), BY4742 pet104::KANMX4 (Euroscarf), and

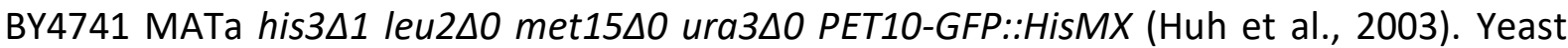
were transformed by standard lithium acetate/polyethylene glycol procedure. Yeast cells expressing different $\mathrm{AH}$ constructs were grown in synthetic complete medium lacking uracil (SC-Ura, $6.7 \mathrm{~g} / \mathrm{l}$ yeast nitrogen base, amino acid supplement without uracil, $2 \%$ glucose). To induce LDs, yeast cells either grown in SC-Ura for $24 \mathrm{~h}$ at $30^{\circ} \mathrm{C}$ (stationary phase) or for $24 \mathrm{~h}$ in SC-Ura, followed by $24 \mathrm{~h}$ incubation in oleic acid $(\mathrm{OA})$ medium $(0.67 \%$ yeast nitrogen base without amino acids, $0.1 \%$ yeast extract, $0.1 \%(\mathrm{v} / \mathrm{v})$ oleate, $0.25 \%(\mathrm{v} / \mathrm{v})$ Tween 40 , amino acid supplement lacking uracil). For imaging of LDs in early stationary phase, yeast cells were inoculated from a preculture and grown at $30^{\circ} \mathrm{C}$ in SC-Ura to a final $\mathrm{OD}_{600}=1-2$.

\section{Cell culture and transfection}

HeLa cells were grown in Dulbecco's modified Eagle's medium (DMEM) supplemented with $4.5 \mathrm{~g} / \mathrm{l}$ glucose (Life technologies), 10\% fetal bovine serum (FBS, Life technology) and 1\% Penicillin/Streptomycin antibiotics (Life technologies). For protein expression, subconfluent cells were transfected with Lipofectamine 2000 (Invitrogen) in Optimem medium (Life technologies) for $6 \mathrm{~h}$, followed by $16 \mathrm{~h}$ in standard growth medium before the cells were fixed and prepared for imaging.

Drosophila S2 cells (ThermoFisher) were cultured in Schneider's Drosophila medium (Invitrogen) supplemented with $10 \% \mathrm{FBS}$ and $1 \%$ Penicillin/Streptomycin at $25^{\circ} \mathrm{C}$. For generating stably-transfected cells, cells were incubated with plasmid DNA and TransIT-Insect Reagent (Mirus), followed by selection with $2 \mu \mathrm{g} / \mathrm{ml}$ puromycin (Life technologies) for 2 weeks. Protein expression from the metal-inducible promoter was induced for $48 \mathrm{~h}$ with the 
addition of $100 \mu \mathrm{M}$ Cu-sulfate to the medium. Lipid droplets were induced with $1 \mathrm{mM}$ oleic acid (Sigma) in complex with fatty-acid free BSA (Sigma) for $24 \mathrm{~h}$. RNAi depletion against CCT1 was performed as described (Copic et al., 2018).

\section{Fluorescent microscopy}

For imaging of purified protein-oil emulsions, emulsions prepared with fluorescent protein were gently mixed in the glass tube before $1.5 \mu \mathrm{l}$ of emulsion was withdrawn with a long 200 $\mu l$ tip and placed on an untreated glass slides (Thermo Scientific). A coverslip was carefully placed on top without applying any pressure.

Yeast cells were harvested by centrifugation, washed, placed on a glass slide and covered with a coverslip. For some experiments, LDs were stained with $1 \mu \mathrm{g} / \mathrm{ml}$ Bodipy 493/503 (Life Technologies) or with Autodot blue dye (Clinisciences) diluted 1000 -fol for $30 \mathrm{~min}$ at room temp, after which the cells were washed twice and imaged. Drosophila S2 cells were imaged on glass slides in the same way as yeast cells.

Transfected HeLa cells were fixed with 3.2\% paraformaldehyde (Sigma) in PBS for 30 min at room temp. After washing three times with PBS, cells were stained with Bodipy 493/503 at 1 $\mu \mathrm{g} / \mathrm{ml}$ for $30 \mathrm{~min}$ at room temperature and washed three times with PBS. Cells were mounted on coverslips with Prolong (Life technologies).

Images of emulsions, yeast and S2 cells were acquired at room temperature with an Axio Observer Z1 (Zeiss) microscope, equipped with an oil immersion plan-Apochromat 100x/1.4 objective, an SCMOS PRIME 95 (Photometrics) camera, and a spinning-disk confocal system CSU-X1 (Yokogawa) driven by MetaMorph software (Molecular Devices). GFP-tagged or Alex488-labeled proteins and mCherry-tagged or Alex568-labeled proteins were visualized with a GFP Filter 535AF45 and an RFP Filter 590DF35, respectively. When imaging emulsions, images were acquired in 10 to $15 \mathrm{z}$-sections of $0.2 \mu \mathrm{m}$ were taken. For imaging HeLa cells and quantification of LD-to-PM signal ratio in yeast, we used an LSM 780 confocal microscope (Zeiss) with a x63/1.4 oil objective and a PMT GaAsP camera, driven by ZEN software. Images were processed with ImageJ and prepared for figures with Canvas Draw (canvas X). 


\section{Fluorescence recovery after photobleaching (FRAP)}

FRAP assays in vitro were performed on freshly-prepared fluorescent emulsions with Alex488labeled proteins on glass slides using the CSU-X1 spinning disc microscope and 100x objective, bleaching laser with a wavelength of $473 \mathrm{~nm}$ and iLas software controlled by Metamorph. Several circular areas of $25 \times 25$ pixels were bleached in each field ( $828 \times 960$ pixels), either on oil particles or in surrounding solution. The following FRAP time-course was used: 6 images pre-bleach, then bleach followed by $10 \mathrm{~s}$ of 1 image/s, $60 \mathrm{~s}$ of 1 image/10 s, and finally $600 \mathrm{~s}$ of 1 image/30 s (or until the loss of focus). Fluorescence of the bleached area at each time point was normalized to the average fluorescence before bleaching. Data was processed using Excel.

For FRAP assays in yeast cells, a circular area of $15 \times 15$ pixels in a cell expressing a GFP-fusion protein was bleached, either on the LDs or on the plasma membrane. 5 images were taken before bleaching, followed by a post-bleach time-course: $15 \mathrm{~s}$ of 1 image/s, $60 \mathrm{~s}$ of 1 image/5 $\mathrm{s}$, and $\sim 200 \mathrm{~s}$ of 1 image/20 s. Background fluorescence outside the cell was subtracted from the bleached area and the signal was normalized to the whole cell signal for each time-point. Data was processed with Excel and plotted using SigmaPlot (Systat Software).

FRAP assays in Drosophila S2 cells expressing Plin4 12mer-GFP were performed as for yeast, except that 3 circular areas of $15 \times 15$ pixels containing isolated LDs were selected per cell. The following FRAP time-course was used: 5 images pre-bleach, then bleach, followed by $30 \mathrm{~s}$ of 1 image/s, 60 s of 1 image/5s, and finally $200 \mathrm{~s}$ of 1 image/20 s. Data was analysed in Excel and plotted using SigmaPlot. To obtain the half-time of recovery, average curves from the 3 FRAP measurements from the same cell were fitted with an exponential-rise equation.

\section{Protein exchange assay on protein-oil emulsions}

Emulsions were prepared as described using unlabeled protein at $0.5 \mathrm{mg} / \mathrm{ml}$ and checked by microscopy using CSU-X1 spinning disc microscope (time 0). Then, fluorescent Plin4 12merAlexa488 was gently added to the suspension to a final concentration of $0.025 \mathrm{mg} / \mathrm{ml}(20: 1$, unlabeled protein : labeled Plin4 12mer). Samples from the emulsions were withdrawn at indicated time-points without mixing and imaged on glass slides. The re-vortex sample was prepared after $2 \mathrm{~h}$ of incubation by withdrawing $20 \mu \mathrm{l}$ of the emulsion and vortexing it in a fresh $600 \mu \mathrm{l}$ glass tube in the same manner as for initial emulsion preparation. Samples were imaged in $10 \mathrm{z}$-sections of $0.5 \mu \mathrm{m}$ in randomly-selected fields of $76 \mu \mathrm{m} \times 101 \mu \mathrm{m}$. The z-section 
containing the highest number of small droplets was selected for analysis.

\section{Image analysis}

Images were analysed using ImageJ/Fiji (Schindelin et al., 2012). To quantify the number of droplets in protein-oil emulsions, the number of particles in a randomly-selected area in a single z-section was counted using 'find maxima' in the fluorescent channel with noise tolerance set to 100. Larger clusters were counted manually. For quantification in the exchange assay, the noise tolerance was set to 150 . To quantify the number of yeast cells with protein signal on LDs, cells were counted manually after applying the same brightness/contrast settings to all images. To quantify the ratio of LD to PM protein signal (mCherry fusions), Pet10-GFP LD marker was used to select the regions of interest (ROIs) corresponding to LDs and the total mCherry fluorescence in the ROls was recorded. For the quantification of PM fluorescence, images were converted to binary to select the whole yeast perimeter. Them, a band of 5 pixels was applied to include all of PM signal. After background subtraction, the total LD signal per cell was divided by the total PM signal.

LD size in yeast cells grown in oleic-acid medium was measured using the fluorescent protein. Isolated LDs were fitted manually with a perfect circle and the size of each circular area was recorder. Data were analysed in Excel and plotted with KaleidaGraph (Synergy software).

To determine the fraction of LDs in HeLa cells that were positive for transfected fluorescent protein, a single z-section that contained the most LDs in a cell was first selected. All LDs in the selected cell section were identified in the green (Bodipy dye) channel using the 'Analyze particle' plug-in. LDs positive for fluorescent protein were then identified by determining a threshold value for the red fluorescent signal (mCherry-protein fusion), 1.4x above average cellular fluorescence, and counting all LDs with fluorescence above this threshold. This number was divided by the total LD number to calculate the fraction of LDs in one cell section positive for protein. Data was processed in Microsoft Excel and plotted using SigmaPlot. 


\section{Acknowledgments}

We thank C. La Torre Garay, M. Tuljak, V. Countremoulins and N. Joly for technical help, and R. Schneiter and S. Léon for plasmids. We acknowledge the IJM ImagoSeine facility, member of IBiSA and the France-Biolmaging infrastructure (ANR-10-INBS-04), and L. Bousset, V. Albanèse, C. Jackson, J.M. D'Ambrosio, R. Gautier, J. Snoj, N. Mejhert, M. Lang, U. Tkalec and members of the Jackson-Verbavatz team for helpful discussions and comments on the manuscript. This work was supported by the CNRS, including a CNRS PICS grant, the Slovenian Research Agency (research core funding No. P1-0055), "Fondation pour la Recherche Médicale", grant number DEQ20150934717, and PhD fellowships from the French "Ministère de I'Education National, de l'Enseignement Supérieur de la Recherche" and Fondation ARC pour la recherche sur le cancer (DOC20190509052) to M.G.-A.

\section{Author Contributions}

Manuel Giménez-Andrés: Conceptualization, Data curation, Formal analysis, Investigation, Methodology, Writing-review and editing

Tadej Emersic: Conceptualization, Data curation, Formal analysis, Investigation, Methodology Sandra Antoine-Bally: Data curation, Formal analysis, Investigation, Methodology Bruno Antonny: Conceptualization, Formal analysis, Writing-review and editing Jure Derganc: Supervision, Conceptualization, Formal analysis, Methodology, Writing-review and editing

Alenka Copic: Supervision, Conceptualization, Data curation, Formal analysis, Investigation, Methodology, Writing-original draft, Funding acquisition

\section{Competing Interests}

The authors have no competing interests to declare. 


\section{References}

Ajjaji, D., K. Ben M'barek, M.L. Mimmack, C. England, H. Herscovitz, L. Dong, R.G. Kay, S. Patel, V. Saudek, D.M. Small, D.B. Savage, and A.R. Thiam. 2019. Dual binding motifs underpin the hierarchical association of perilipins1-3 with lipid droplets. Mol Biol Cell. 30:703-716. doi:10.1091/mbc.E18-08-0534.

Bacle, A., R. Gautier, C.L. Jackson, P.F.J. Fuchs, and S. Vanni. 2017. Interdigitation between Triglycerides and Lipids Modulates Surface Properties of Lipid Droplets. Biophys J. 112:14171430. doi:10.1016/j.bpj.2017.02.032.

Ben M'barek, K., D. Ajjaji, A. Chorlay, S. Vanni, L. Forêt, and A.R. Thiam. 2017. ER Membrane Phospholipids and Surface Tension Control Cellular Lipid Droplet Formation. Dev Cell. 1-31. doi:10.1016/j.devcel.2017.05.012.

Bersuker, K., and J.A. Olzmann. 2017. Establishing the lipid droplet proteome_Mechanisms of lipid droplet protein targeting and degradation. BBA - Mol Cell Biol Lipids. 1862:1166-1177. doi:10.1016/j.bbalip.2017.06.006.

Bersuker, K., C.W.H. Peterson, M. To, S.J. Sahl, V. Savikhin, E.A. Grossman, D.K. Nomura, and J.A. Olzmann. 2018. A Proximity Labeling Strategy Provides Insights into the Composition and Dynamics of Lipid Droplet Proteomes. Dev Cell. 44:97-112.e7. doi:10.1016/j.devcel.2017.11.020.

Bibow, S., Y. Polyhach, C. Eichmann, C.N. Chi, J. Kowal, S. Albiez, R.A. McLeod, H. Stahlberg, G. Jeschke, P. Güntert, and R. Riek. 2017. Solution structure of discoidal high-density lipoprotein particles with a shortened apolipoprotein A-I. Nat Struct Mol Biol. 24:187-193. doi:10.1038/nsmb.3345.

Biegert, A., and J. Söding. 2008. De novo identification of highly diverged protein repeats by probabilistic consistency. Bioinformatics. 24:807-814. doi:10.1093/bioinformatics/btn039.

Bininda-Emonds, O. R. P., Cardillo, M., Jones, K. E., MacPhee, R. D. E., Beck, R. M. D., Grenyer, R., Price, S. A., Vos, R. A., Gittleman, J. L. and Purvis, A. 2007. The delayed rise of present-day mammals. Nature 446: 507-512. doi:10.1038/nature05634.

Blanchette-Mackie, E.J., N.K. Dwyer, T. Barber, R.A. Coxey, T. Takeda, C.M. Rondinone, J.L. Theodorakis, A.S. Greenberg, and C. Londos. 1995. Perilipin is located on the surface layer of intracellular lipid droplets in adipocytes. J Lipid Res. 36:1211-1226.

Bouvet, S., Golinelli-Cohen, M., Contremoulins, V., Jackson, C.L. 2013. Targeting of the Arf-GEF GBF1 to lipid droplets and Golgi membranes. J Cell Sci 126:4794-4805.

Brasaemle, D.L., G. Dolios, L. Shapiro, and R. Wang. 2004. Proteomic analysis of proteins associated with lipid droplets of basal and lipolytically stimulated 3T3-L1 adipocytes. J Biol Chem. 279:46835-46842. doi:10.1074/jbc.M409340200.

Bulankina, A.V., A. Deggerich, D. Wenzel, K. Mutenda, J.G. Wittmann, M.G. Rudolph, K.N. Burger, and S. Honing. 2009. TIP47 functions in the biogenesis of lipid droplets. J Cell Biol. 185:641-655. doi:10.1074/jbc.M402264200.

Bussell, R., Jr, and D. Eliezer. 2003. A Structural and Functional Role for 11-mer Repeats in $\alpha$ Synuclein and Other Exchangeable Lipid Binding Proteins. J Mol Biol. 329:763-778. doi:10.1016/S0022-2836(03)00520-5. 
Chen, W., B. Chang, X. Wu, L. Li, M. Sleeman, and L. Chan. 2013. Inactivation of Plin4 downregulates Plin5 and reduces cardiac lipid accumulation in mice. Am J Physiol Endocrinol Metab. 304:E7709. doi:10.1152/ajpendo.00523.2012.

Chorlay, A., L. Monticelli, J.V. Ferreira, K. Ben M'barek, D. Ajjaji, S. Wang, E. Johnson, R. Beck, M. Omrane, M. Beller, P. Carvalho, and A.R. Thiam. 2019. Membrane Asymmetry Imposes Directionality on Lipid Droplet Emergence from the ER. Dev Cell. 1-26. doi:10.1016/j.devcel.2019.05.003.

Copic, A., S. Antoine-Bally, M. Giménez-Andrés, C. La Torre Garay, B. Antonny, M.M. Manni, S. Pagnotta, J. Guihot, and C.L. Jackson. 2018. A giant amphipathic helix from a perilipin that is adapted for coating lipid droplets. Nat Commun. 9:1332. doi:10.1038/s41467-018-03717-8.

Crooks, G.E., G. Hon, J.-M. Chandonia, and S.E. Brenner. 2004. WebLogo: a sequence logo generator. Genome Res. 14:1188-1190. doi:10.1101/gr.849004.

Faini, M., R. Beck, F.T. Wieland, and J.A.G. Briggs. 2013. Vesicle coats: structure, function, and general principles of assembly. Trends Cell Biol. 1-10. doi:10.1016/j.tcb.2013.01.005.

Gao, Q., D.D. Binns, L.N. Kinch, N.V. Grishin, N. Ortiz, X. Chen, and J.M. Goodman. 2017. Pet10p is a yeast perilipin that stabilizes lipid droplets and promotes their assembly. J Cell Biol. 181:jcb.201610013-23. doi:10.1083/jcb.201610013.

Gautier, R., D. Douguet, B. Antonny, and G. Drin. 2008. HELIQUEST: a web server to screen sequences with specific -helical properties. Bioinformatics. 24:2101-2102. doi:10.1093/bioinformatics/btn392.

Giménez-Andrés, M., A. Copic, and B. Antonny. 2018. The Many Faces of Amphipathic Helices. Biomolecules. 8:45. doi:10.3390/biom8030045.

Granneman, J.G., V.A. Kimler, H. Zhang, X. Ye, X. Luo, J.H. Postlethwait, and R. Thummel. 2017. Lipid droplet biology and evolution illuminated by the characterization of a novel perilipin in teleost fish. Elife. 6:799. doi:10.7554/eLife.21771.

Guo, Y., T.C. Walther, M. Rao, N. Stuurman, G. Goshima, K. Terayama, J.S. Wong, R.D. Vale, P. Walter, and R.V. Farese. 2008. Functional genomic screen reveals genes involved in lipid-droplet formation and utilization. Nature. 453:657-661. doi:10.1038/nature06928.

Hansen, J.S., S.M. x000E9, H.A. Jones, O.G.X. ransson, and K. Lindkvist-Petersson. 2017. Visualization of lipid directed dynamics of perilipin 1 in human primary adipocytes. Sci Rep. 1-14. doi:10.1038/s41598-017-15059-4.

Hickenbottom, S.J., A.R. Kimmel, C. Londos, and J.H. Hurley. 2004. Structure of a lipid droplet protein; the PAT family member TIP47. Structure. 12:1199-1207. doi:10.1016/j.str.2004.04.021.

Hsieh, K., Y.K. Lee, C. Londos, B.M. Raaka, K.T. Dalen, and A.R. Kimmel. 2012. Perilipin family members preferentially sequester to either triacylglycerol-specific or cholesteryl-ester-specific intracellular lipid storage droplets. J Cell Sci. 125:4067-4076. doi:10.1242/jcs.104943.

Huh, W.-K., J.V. Falvo, L.C. Gerke, A.S. Carroll, R.W. Howson, J.S. Weissman, and E.K. O'Shea. 2003. Global analysis of protein localization in budding yeast. Nature. 425:686-691. doi:10.1038/nature02026. 
Jacquier, N., S. Mishra, V. Choudhary, and R. Schneiter. 2013. Expression of oleosin and perilipins in yeast promotes formation of lipid droplets from the endoplasmic reticulum. J Cell Sci. 126:51985209. doi:10.1242/jcs.131896.

Jao, C.C., B.G. Hegde, J. Chen, I.S. Haworth, and R. Langen. 2008. Structure of membrane-bound alpha-synuclein from site-directed spin labeling and computational refinement. Proc Natl Acad Sci USA. 105:19666-19671. doi:10.1073/pnas.0807826105.

Kory, N., A.R. Thiam, R.V. Farese Jr, and T.C. Walther. 2015. Protein Crowding Is a Determinant of Lipid Droplet Protein Composition. Dev Cell. 34:351-363. doi:10.1016/j.devcel.2015.06.007.

Krahmer, N., Y. Guo, F. Wilfling, M. Hilger, S. Lingrell, K. Heger, H.W. Newman, M. Schmidt-Supprian, D.E. Vance, M. Mann, R.V.F. Jr, and T.C. Walther. 2011. Phosphatidylcholine Synthesis for Lipid Droplet Expansion Is Mediated by Localized Activation of CTP:Phosphocholine Cytidylyltransferase. Cell Metab. 14:504-515. doi:10.1016/j.cmet.2011.07.013.

Lord, S.J., K.B. Velle, R.D. Mullins, and L.K. Fritz-Laylin. 2020. SuperPlots: Communicating reproducibility and variability in cell biology. J Cell Biol. 219:94-10. doi:10.1083/jcb.202001064.

Lundquist, P.K., K.-K. Shivaiah, and R. Espinoza-Corral. 2020. Lipid droplets throughout the evolutionary tree. Prog Lipid Res. 78:101029. doi:10.1016/j.plipres.2020.101029.

McClements, D.J., and C.E. Gumus. 2016. Natural emulsifiers - Biosurfactants, phospholipids, biopolymers, and colloidal particles: Molecular and physicochemical basis of functional performance. Adv Colloid Interface Sci. 234:3-26. doi:10.1016/j.cis.2016.03.002.

McManaman, J.L., W. Zabaronick, J. Schaack, and D.J. Orlicky. 2003. Lipid droplet targeting domains of adipophilin. J Lipid Res. 44:668-673. doi:10.1194/jIr.C200021-JLR200.

Mejhert, N., L. Kuruvilla, K.R. Gabriel, S.D. Elliott, M.-A. Guie, H. Wang, Z.W. Lai, E.A. Lane, R. Christiano, N.N. Danial, R.V. Farese Jr, and T.C. Walther. 2020. Partitioning of MLX-Family Transcription Factors to Lipid Droplets Regulates Metabolic Gene Expression. Mol Cell. 77:12511264.e9. doi:10.1016/j.molcel.2020.01.014.

Melchior, J.T., R.G. Walker, A.L. Cooke, J. Morris, M. Castleberry, T.B. Thompson, M.K. Jones, H.D. Song, K.-A. Rye, M.N. Oda, M.G. Sorci-Thomas, M.J. Thomas, J.W. Heinecke, X. Mei, D. Atkinson, J.P. Segrest, S. Lund-Katz, M.C. Phillips, and W.S. Davidson. 2017. A consensus model of human apolipoprotein A-I in its monomeric and lipid-free state. Nat Struct Mol Biol. 24:1093-1099. doi:10.1038/nsmb.3501.

Mirheydari, M., S.S. Rathnayake, H. Frederick, T. Arhar, E.K. Mann, S. Cocklin, and E.E. Kooijman. 2016. Insertion of perilipin 3 into a glycero(phospho)lipid monolayer depends on lipid headgroup and acyl chain species. J Lipid Res. 57:1465-1476. doi:10.1194/jlr.M068205.

Miura, S., J.-W. Gan, J. Brzostowski, M.J. Parisi, C.J. Schultz, C. Londos, B. Oliver, and A.R. Kimmel. 2002. Functional Conservation for Lipid Storage Droplet Association among Perilipin, ADRP, and TIP47 (PAT)-related Proteins in Mammals, Drosophila, and Dictyostelium. J Biol Chem. 277:32253-32257. doi:10.1074/jbc. M204410200.

Nakamura, N., and T. Fujimoto. 2003. Adipose differentiation-related protein has two independent domains for targeting to lipid droplets. Biochem Biophys Res Commun. 306:333-338. doi:10.1016/S0006-291X(03)00979-3. 
Nakamura, N., T. Akashi, T. Taneda, H. Kogo, A. Kikuchi, and T. Fujimoto. 2004. ADRP is dissociated from lipid droplets by ARF1-dependent mechanism. Biochem Biophys Res Commun. 322:957965. doi:10.1016/j.bbrc.2004.08.010.

Ohsaki, Y., M. Suzuki, and T. Fujimoto. 2014. Open Questions in Lipid Droplet Biology. Chemistry \& Biology. 21:86-96. doi:10.1016/j.chembiol.2013.08.009.

Olzmann, J.A., and P. Carvalho. 2019. Dynamics and functions of lipid droplets. Nat Rev Mol Cell Biol. 1-19. doi:10.1038/s41580-018-0085-z.

Osterman, N., J. Derganc, and D. Svenšek. 2016. Formation of vortices in long microcavities at low Reynolds number. Microfluidics and Nanofluidics. 20:33.

Pace, C.N., and J.M. Scholtz. 1998. A helix propensity scale based on experimental studies of peptides and proteins. Biophys j. 75:422-427.

Pataki, C.I., J. Rodrigues, L. Zhang, J. Qian, B. Efron, T. Hastie, J.E. Elias, M. Levitt, and R.R. Kopito. 2018. Proteomic analysis of monolayer-integrated proteins on lipid droplets identifies amphipathic interfacial $\alpha$-helical membrane anchors. Proc Natl Acad Sci USA. 115:E8172-E8180. doi:10.1073/pnas.1807981115.

Phillips, M.C. 2013. New insights into the determination of HDL structure by apolipoproteins: Thematic Review Series: High Density Lipoprotein Structure, Function, and Metabolism. J Lipid Res. 54:2034-2048. doi:10.1194/jlr.R034025.

Pourmousa, M., H.D. Song, Y. He, J.W. Heinecke, J.P. Segrest, and R.W. Pastor. 2018. Tertiary structure of apolipoprotein A-I in nascent high-density lipoproteins. Proc Natl Acad Sci USA. 115:5163-5168. doi:10.1073/pnas.1721181115.

Prévost, C., M.E. Sharp, N. Kory, Q. Lin, G.A. Voth, R.V. Farese, and T.C. Walther. 2018. Mechanism and Determinants of Amphipathic Helix-Containing Protein Targeting to Lipid Droplets. Dev Cell. 44:73-86.e4. doi:10.1016/j.devcel.2017.12.011.

Rowe, E.R., M.L. Mimmack, A.D. Barbosa, A. Haider, I. Isaac, M.M. Ouberai, A.R. Thiam, S. Patel, V. Saudek, S. Siniossoglou, and D.B. Savage. 2016. Conserved Amphipathic Helices Mediate Lipid Droplet Targeting of Perilipins 1-3. J Biol Chem. 291:6664-6678. doi:10.1074/jbc.M115.691048.

Ruggieri, A., S. Naumenko, M.A. Smith, E. Iannibelli, F. Blasevich, C. Bragato, S. Gibertini, K. Barton, M. Vorgerd, K. Marcus, P. Wang, L. Maggi, R. Mantegazza, J.J. Dowling, R.A. Kley, M. Mora, and B.A. Minassian. 2020. Multiomic elucidation of a coding 99-mer repeat-expansion skeletal muscle disease. Acta Neuropathologica. 1-5. doi:10.1007/s00401-020-02164-4.

Saito, H., P. Dhanasekaran, D. Nguyen, P. Holvoet, S. Lund-Katz, and M.C. Phillips. 2003. Domain Structure and Lipid Interaction in Human Apolipoproteins A-I and E, a General Model. J Biol Chem. 278:23227-23232. doi:10.1074/jbc.M303365200.

Saleem, M., S. Morlot, A. Hohendahl, J. Manzi, M. Lenz, and A. Roux. 2015. A balance between membrane elasticity and polymerization energy sets the shape of spherical clathrin coats. Nat Commun. 6:6249. doi:10.1038/ncomms7249.

Santinho, A., V.T. Salo, A. Chorlay, S. Li, X. Zhou, M. Omrane, E. Ikonen, and A.R. Thiam. 2020. Membrane Curvature Catalyzes Lipid Droplet Assembly. Curr Biol. 1-21. doi:10.1016/j.cub.2020.04.066. 
Schägger, H., and G. von Jagow. 1987. Tricine-sodium dodecyl sulfate-polyacrylamide gel electrophoresis for the separation of proteins in the range from 1 to $100 \mathrm{kDa}$. Anal Biochem. 166:368-379.

Schekman, R., and L. Orci. 1996. Coat proteins and vesicle budding. Science. 271:1526-1533.

Scherer, P.E., P.E. Bickel, M. Kotler, and H.F. Lodish. 1998. Cloning of cell-specific secreted and surface proteins by subtractive antibody screening. Nat Biotechnol. 16:581-586. doi:10.1038/nbt0698-581.

Schindelin, J., I. Arganda-Carreras, E. Frise, V. Kaynig, M. Longair, T. Pietzsch, S. Preibisch, C. Rueden, S. Saalfeld, B. Schmid, J.-Y. Tinevez, D.J. White, V. Hartenstein, K. Eliceiri, P. Tomancak, and A. Cardona. 2012. Fiji: an open-source platform for biological-image analysis. Nat Meth. 9:676-682. doi:10.1038/nmeth.2019.

Soni, K.G., G.A. Mardones, R. Sougrat, E. Smirnova, C.L. Jackson, and J.S. Bonifacino. 2009. Coatomerdependent protein delivery to lipid droplets. J Cell Sci. 122:1834-1841. doi:10.1242/jcs.045849.

Sorre, B., A. Callan-Jones, J. Manzi, B. Goud, J. Prost, P. Bassereau, and A. Roux. 2012. Nature of curvature coupling of amphiphysin with membranes depends on its bound density. Proc Natl Acad Sci USA. 109:173-178. doi:10.1073/pnas.1103594108.

Stenkula, K.G., and C. Erlanson-Albertsson. 2018. Adipose cell size: importance in health and disease. Am J Physiol Regul Integr Comp Physiol. 315:R284-R295. doi:10.1152/ajpregu.00257.2017.

Subramanian, V., A. Garcia, A. Sekowski, and D.L. Brasaemle. 2004. Hydrophobic sequences target and anchor perilipin A to lipid droplets. J Lipid Res. 45:1983-1991. doi:10.1194/jlr.M400291JLR200.

Sztalryd, C., and D.L. Brasaemle. 2017. The perilipin family of lipid droplet proteins: Gatekeepers of intracellular lipolysis. BBA - Mol Cell Biol Lipids. 1862:1221-1232. doi:10.1016/j.bbalip.2017.07.009.

Targett-Adams, P., D. Chambers, S. Gledhill, R.G. Hope, J.F. Coy, A. Girod, and J. McLauchlan. 2003. Live Cell Analysis and Targeting of the Lipid Droplet-binding Adipocyte Differentiation-related Protein. J Biol Chem. 278:15998-16007. doi:10.1074/jbc.M211289200.

Taylor, M.J., D. Perrais, and C.J. Merrifield. 2011. A High Precision Survey of the Molecular Dynamics of Mammalian Clathrin-Mediated Endocytosis. PLoS Biol. 9:e1000604.

doi:10.1371/journal.pbio.1000604.s013.

Thiam, A.R., B. Antonny, J. Wang, J. Delacotte, F. Wilfling, T.C. Walther, R. Beck, J.E. Rothman, and F. Pincet. 2013a. COPI buds 60-nm lipid droplets from reconstituted water-phospholipidtriacylglyceride interfaces, suggesting a tension clamp function. Proc Natl Acad Sci USA. 110:13244-13249. doi:10.1073/pnas.1307685110.

Thiam, A.R., R.V. Farese Jr, and T.C. Walther. 2013b. The biophysics and cell biology of lipid droplets. Nat Rev Mol Cell Biol. 14:775-786. doi:10.1038/nrm3699.

Vrhovec, S., M. Mally, B. Kavčič, and J. Derganc. 2011. A microfluidic diffusion chamber for reversible environmental changes around flaccid lipid vesicles. Lab Chip. 11:4200-4206.

doi:10.1039/c1lc20531e. 
Wilfling, F., A.R. Thiam, M.-J. Olarte, J. Wang, R. Beck, T.J. Gould, E.S. Allgeyer, F. Pincet, J. Bewersdorf, R.V. Farese, and T.C. Walther. 2014. Arf1/COPI machinery acts directly on lipid droplets and enables their connection to the ER for protein targeting. Elife. 3:e01607. doi:10.7554/elife.01607.

Wilfling, F., H. Wang, J.T. Haas, N. Krahmer, T.J. Gould, A. Uchida, J.-X. Cheng, M. Graham, R. Christiano, F. Fröhlich, X. Liu, K.K. Buhman, R.A. Coleman, J. Bewersdorf, R.V. Farese, and T.C. Walther. 2013. Triacylglycerol synthesis enzymes mediate lipid droplet growth by relocalizing from the ER to lipid droplets. Dev Cell. 24:384-399. doi:10.1016/j.devcel.2013.01.013.

Wolins, N.E., B.K. Quaynor, J.R. Skinner, A. Tzekov, M.A. Croce, M.C. Gropler, V. Varma, A. YaoBorengasser, N. Rasouli, P.A. Kern, B.N. Finck, and P.E. Bickel. 2006. OXPAT/PAT-1 Is a PPARInduced Lipid Droplet Protein That Promotes Fatty Acid Utilization. Diabetes. 55:3418-3428. doi:10.2337/db06-0399.

Wolins, N.E., B.K. Quaynor, J.R. Skinner, M.J. Schoenfish, A. Tzekov, and P.E. Bickel. 2005. S3-12, Adipophilin, and TIP47 package lipid in adipocytes. J Biol Chem. 280:19146-19155. doi:10.1074/jbc.M500978200.

Wolins, N.E., J.R. Skinner, M.J. Schoenfish, A. Tzekov, K.G. Bensch, and P.E. Bickel. 2003. Adipocyte protein S3-12 coats nascent lipid droplets. J Biol Chem. 278:37713-37721. doi:10.1074/jbc.M304025200.

Zechner, R., F. Madeo, and D. Kratky. 2017. Cytosolic lipolysis and lipophagy: two sides of the same coin. Nat Rev Mol Cell Biol. 18:671-684. doi:10.1038/nrm.2017.76.

Zimmermann, L., A. Stephens, S.-Z. Nam, D. Rau, J. Kübler, M. Lozajic, F. Gabler, J. Söding, A.N. Lupas, and V. Alva. 2018. A Completely Reimplemented MPI Bioinformatics Toolkit with a New HHpred Server at its Core. J Mol Biol. 430:2237-2243. doi:10.1016/j.jmb.2017.12.007. 


\section{Figure 1}

A

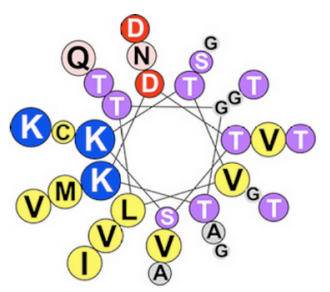

Plin4 AH
C

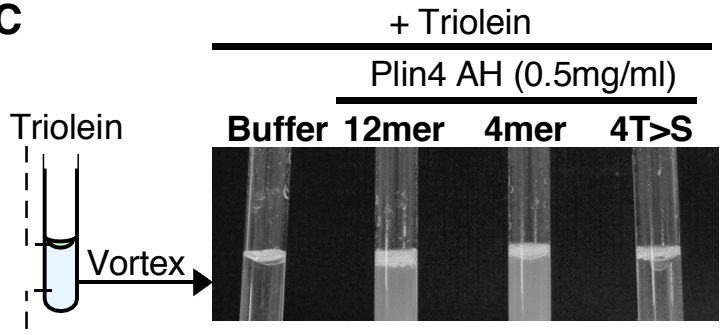

Protein solution

D

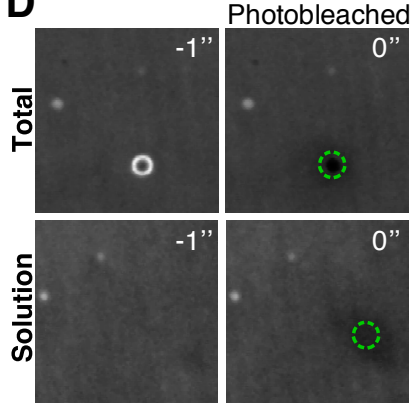

Photobleached
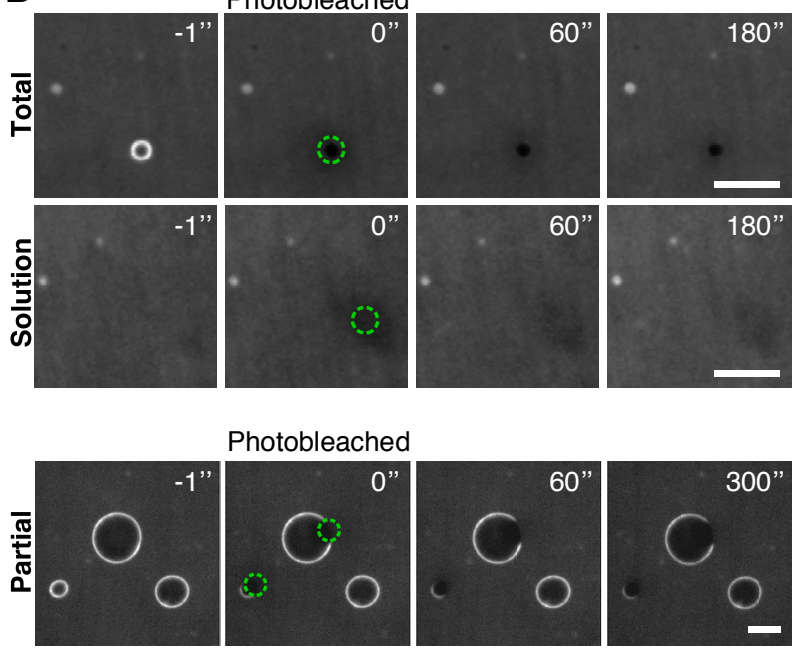

300

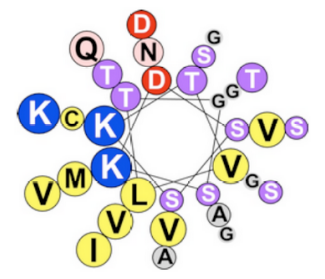

4T $>S$
B

Localization of Plin4-mCherry constructs in

HeLa cells (data from Copic et al., 2018)

\begin{tabular}{|c|c|c|}
\hline $\begin{array}{c}\text { Plin4 } \\
\text { construct }\end{array}$ & $\begin{array}{c}\text { Length } \\
\text { [aa] }\end{array}$ & $\begin{array}{c}\text { \%LDs with protein } \\
\text { / cell [mean } \pm \text { SE] }\end{array}$ \\
\hline 2mer & 64 & 0 \\
\hline 4mer & 132 & $29 \pm 4$ \\
\hline 8mer & 264 & $49 \pm 4$ \\
\hline 12mer & 396 & 100 \\
\hline 20mer & 660 & 100 \\
\hline 4T>S & 132 & 0 \\
\hline $\mathbf{1 T}>\mathbf{V}$ & 132 & 100 \\
\hline
\end{tabular}

돌
E

Plin4 12mer

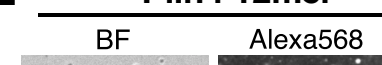

동
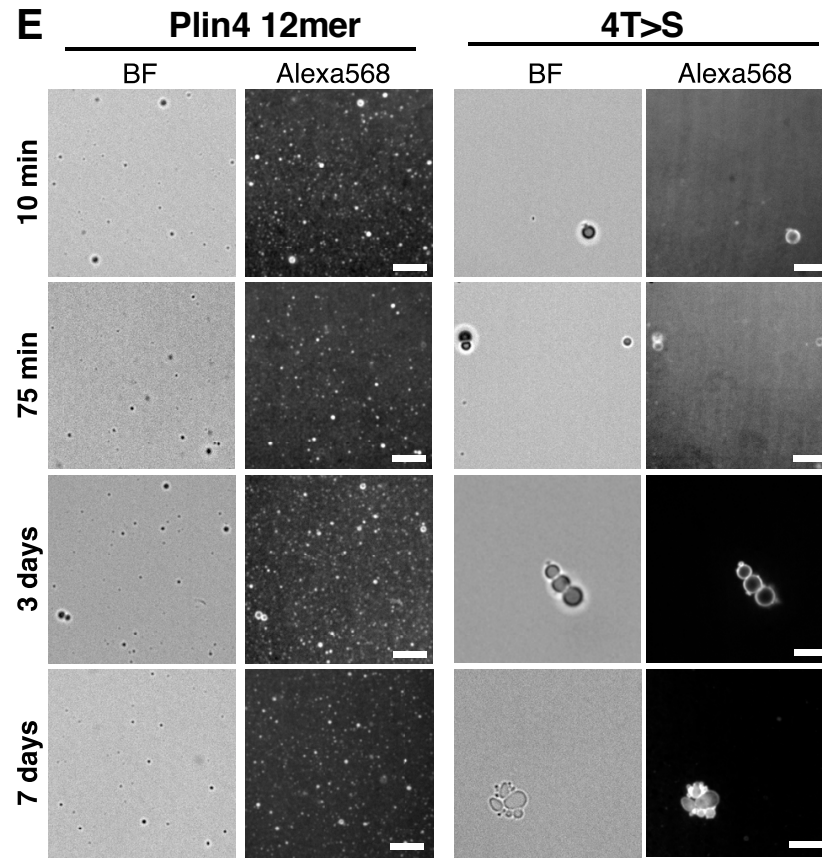

$\mathbf{F}$

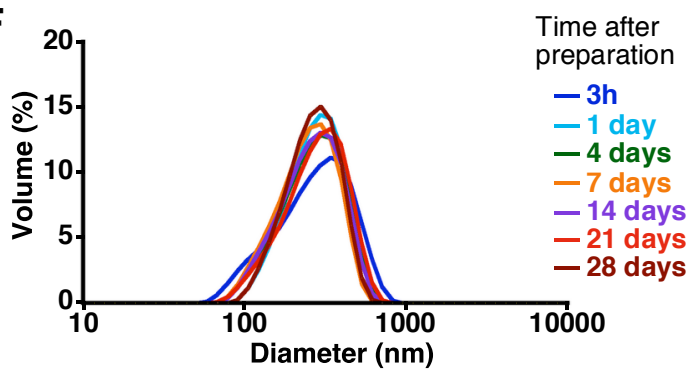

Figure 1: Plin4 AH forms very stable oil particles. A: Helical wheel representations of Plin4 and Plin4 4T>S AHs. B. Summary of LD localization of Plin4 AH and mutants (Copic et al., 2018). C. A $10 \mu \mathrm{l}$ drop of triolein was added to $190 \mathrm{I}$ of HK buffer containing the indicated proteins (Plin4 12mer, Plin4 4mer or $4 \mathrm{~T}>\mathrm{S}$, all at $0.5 \mathrm{mg} / \mathrm{ml}$ ). After vigorous vortexing, the samples were photographed. D. Dynamics of Plin4 12mer interaction with oil as assessed by FRAP (Fluorescence recovery after photobleaching) assays. An emulsion of triolein with unlabeled Plin4 12mer $(0.5 \mathrm{mg} / \mathrm{ml})$ and Alexa488-labeled Plin4 $(0.025 \mathrm{mg} / \mathrm{ml}) 12 \mathrm{mer}$ was prepared as in $\mathrm{C}$ and visualized by fluorescence microscopy. FRAP was performed on large droplets, which were entirely bleached (top row), or in the bulk as a control (middle row). The lower row shows a FRAP experiment performed on a limited region of the surface of the droplet. E. Light microscopy images of Plin4 12mer and 4T>S emulsions at different time points after preparation by vortexing, showing the stability of the particles in the emulsions over several days. F. Size distribution as assessed by dynamic light scattering (DLS) of the particles present in a Plin4 12mer/triolein emulsion from 3 hours to 28 days after the vortexing reaction. Scale bars: $5 \mu \mathrm{m}$. 


\section{Figure 2}

A
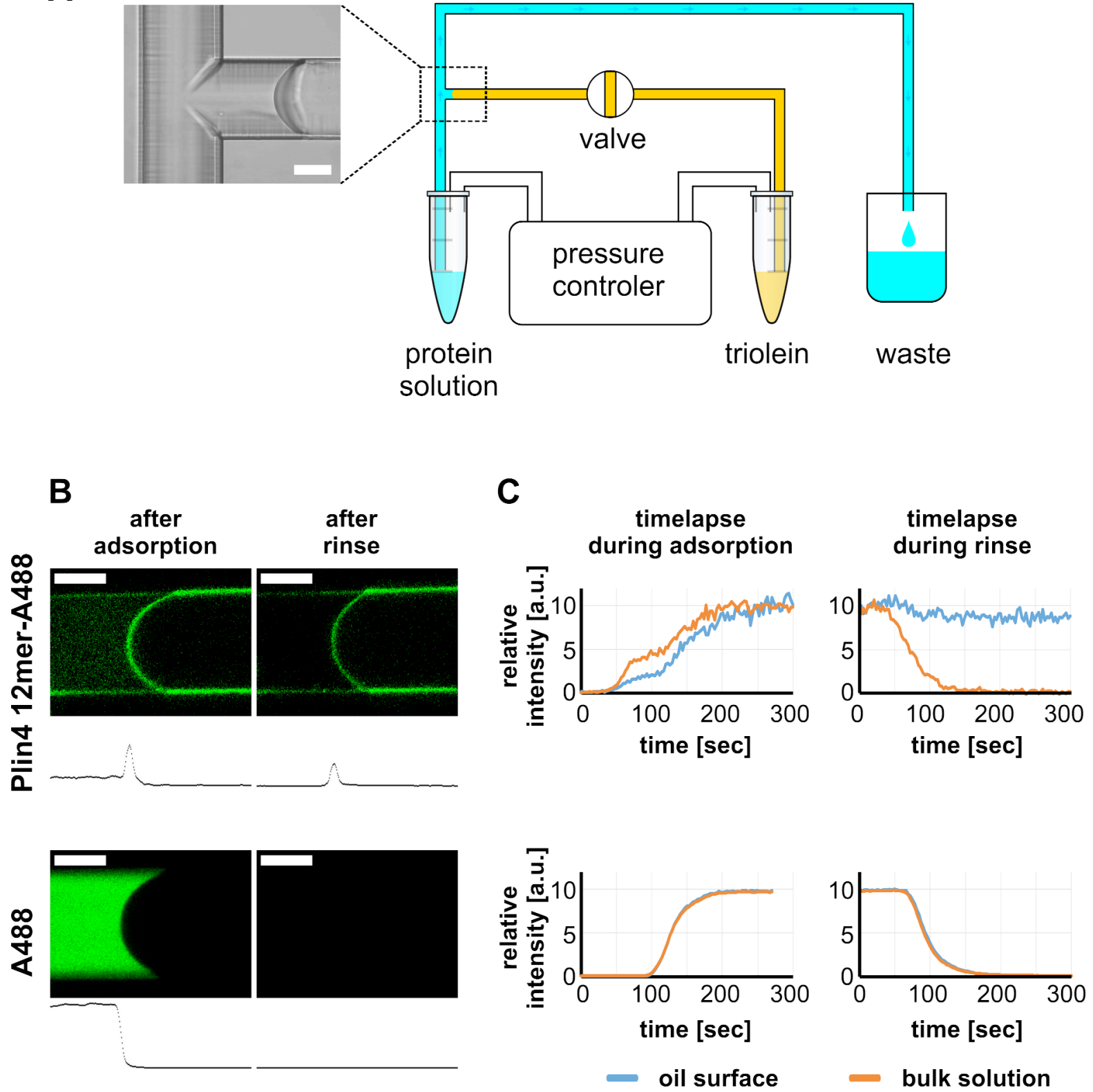

Figure 2. Real-time monitoring of protein-oil interaction in a microfluidic system shows irreversible adsorption of Plin4 12mer-A488 on triolein. A. Scheme of the microfluidics experimental set-up. B. Top row: confocal images of the triolein-buffer interface as formed in the microfluidic system after adsorption of Alexa488-labeled Plin4 12mer on the triolein surface and after rinsing with buffer. Bottom row: control experiment with the free fluorophore Alexa488. The intensity profile along the channel center is shown below each confocal image. The protein adsorbs irreversibly at the oil surface, whereas Alexa488 conjugated to free cysteine (A488) does not. See also supplementary movies 1-4. C. Time course of the signal of Alexa488-labeled Plin4 12mer or of free Alexa-488 in the side channel as quantified from the experiment shown in B. 
Figure 3

A

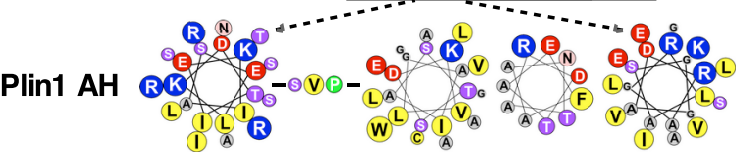

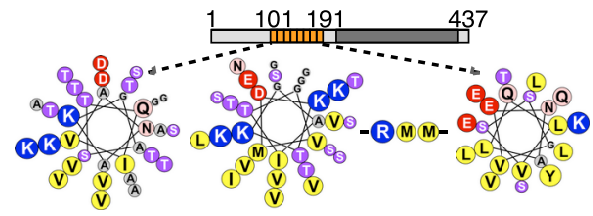

Plin3 AH
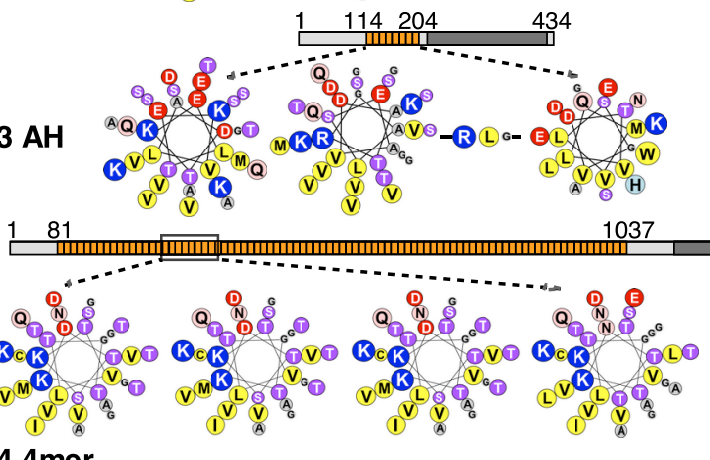

Plin4 4mer

C
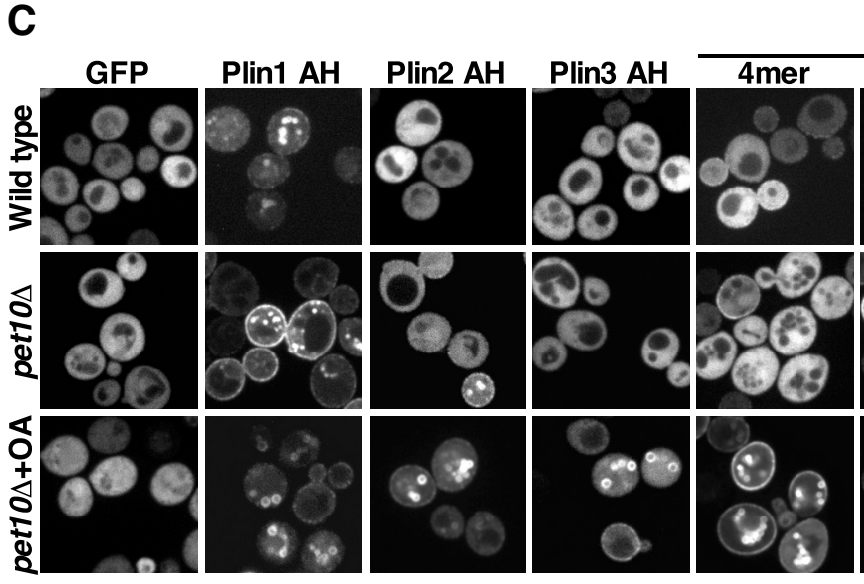

Plin4
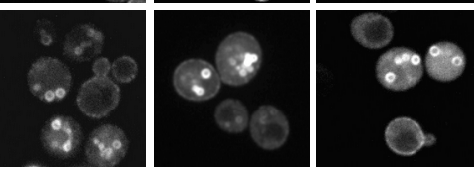

E
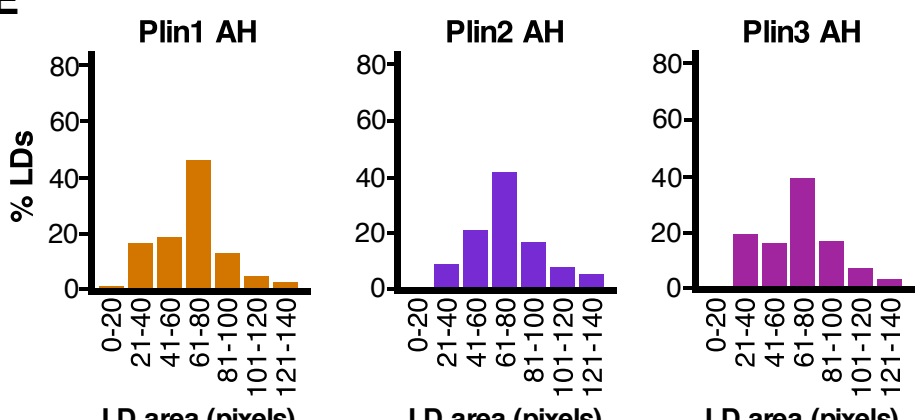

LD area (pixels)
B

\begin{tabular}{|c|c|c|c|c|c|c|}
\hline aa & Plin1 & Plin2 & Plin3 & Plin4 & Plin5 & $\begin{array}{c}\text { Av. } \\
\text { vert }\end{array}$ \\
\hline Trp + Phe & 2.4 & 0 & 1.1 & 0.2 & 3.6 & 5.3 \\
\hline Leu + lle & 18.1 & 9.9 & 7.7 & 6.7 & 8.9 & 11.4 \\
\hline Val & 6 & 15.4 & 16.5 & 15.0 & 23.3 & 6.8 \\
\hline Gly & 8.4 & 9.9 & 9.9 & 15.6 & 3.6 & 7.4 \\
\hline Ala & 18.1 & 9.9 & 8.8 & 10.8 & 7.1 & 7.4 \\
\hline Thr & 6 & 14.3 & 8.8 & 21.3 & 5.4 & 6.2 \\
\hline Ser & 10.8 & 12.1 & 13.2 & 5.4 & 14.3 & 8.1 \\
\hline Asn + Gln & 2.4 & 6.6 & 6.6 & 6.5 & 0 & 10.2 \\
\hline Lys & 4.8 & 8.8 & 7.7 & 8.8 & 5.4 & 7.2 \\
\hline Arg & 7.2 & 1.1 & 2.2 & 0.4 & 8.9 & 4.2 \\
\hline Glu & 7.2 & 4.4 & 6.6 & 0.3 & 8.9 & 3.7 \\
\hline Asp & 4.8 & 3.3 & 6.6 & 5.3 & 7.1 & 5.9 \\
\hline $\begin{array}{c}\text { AH length } \\
\text { (aa) }\end{array}$ & $\mathbf{8 3}$ & $\mathbf{9 1}$ & $\mathbf{9 1}$ & $\mathbf{9 5 7}$ & $\mathbf{5 6}$ & \multicolumn{3}{|c}{} \\
\cline { 1 - 4 } & & & & \multicolumn{3}{|c}{}
\end{tabular}
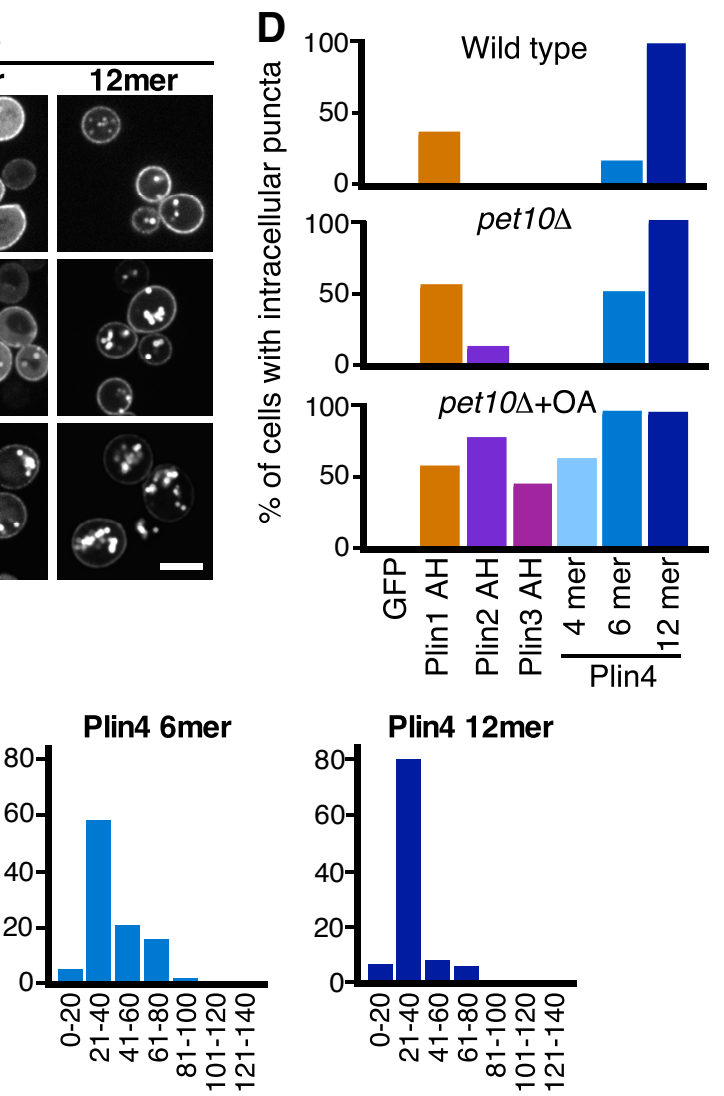

LD area (pixels)

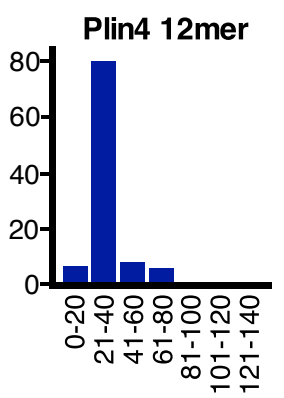

LD area (pixels) 
Figure 3. Comparison of the LD binding properties of the AH of Plin1, 2, 3 and 4 in yeast. A. Helical wheel representation of the AHs of Plin1 (aa 110-189 aa), Plin2 (aa 101-191 aa), Plin3 (aa 114-204) and Plin4 (aa 246-377, corresponding to the Plin4 4mer construct). In the case of Plin1, Plin2 and Plin3, the predicted AH regions are interspersed by short aa linkers, which are also indicated. Diagrams above the helical wheels show the full-length proteins, with $\mathrm{AH}$ regions shown in orange and the 4-helix bundle in dark grey. B. AA composition of the $\mathrm{AH}$ of Plin1, 2, 3 and 4 (in \%) in comparison with the average aa composition of vertebrate proteins (av. vert). The blue and red backgrounds indicate lower or higher \% as compared to vertebrate values, respectively. C. Localization of GFP fusions with the AH region of Plin1, Plin2, Plin3 or Plin4 in S. cerevisiae cells. The experiment was performed with wild-type yeast cells (upper row) or with pet10 $\Delta$ cells (medium row) grown for $24 \mathrm{~h}$ to stationary phase, or with pet10 $\Delta$ cells grown to stationary phase and then transferred for $24 \mathrm{~h}$ to oleic acid (OA) medium (lower row). D. Bar plots of the percentage of yeast cells showing intracellular puncta for the different proteins expressed. 60 cells per each condition were counted in one of at least two representative experiments. E. Quantification of the size distribution of fluorescent LDs (labeled with GFP-fusion proteins) in pet10 + OA cells. The plots show representative measurements from two independent experiments, where the following number of LDs was counted: Plin1 AH, 136; Plin2 AH, 143; Plin3 AH, 133; Plin4 6mer, 148; Plin4 12mer, 159. Pixel size: $0.091 \mu \mathrm{m} \times 0.091 \mu \mathrm{m}$. 


\section{Figure 4}
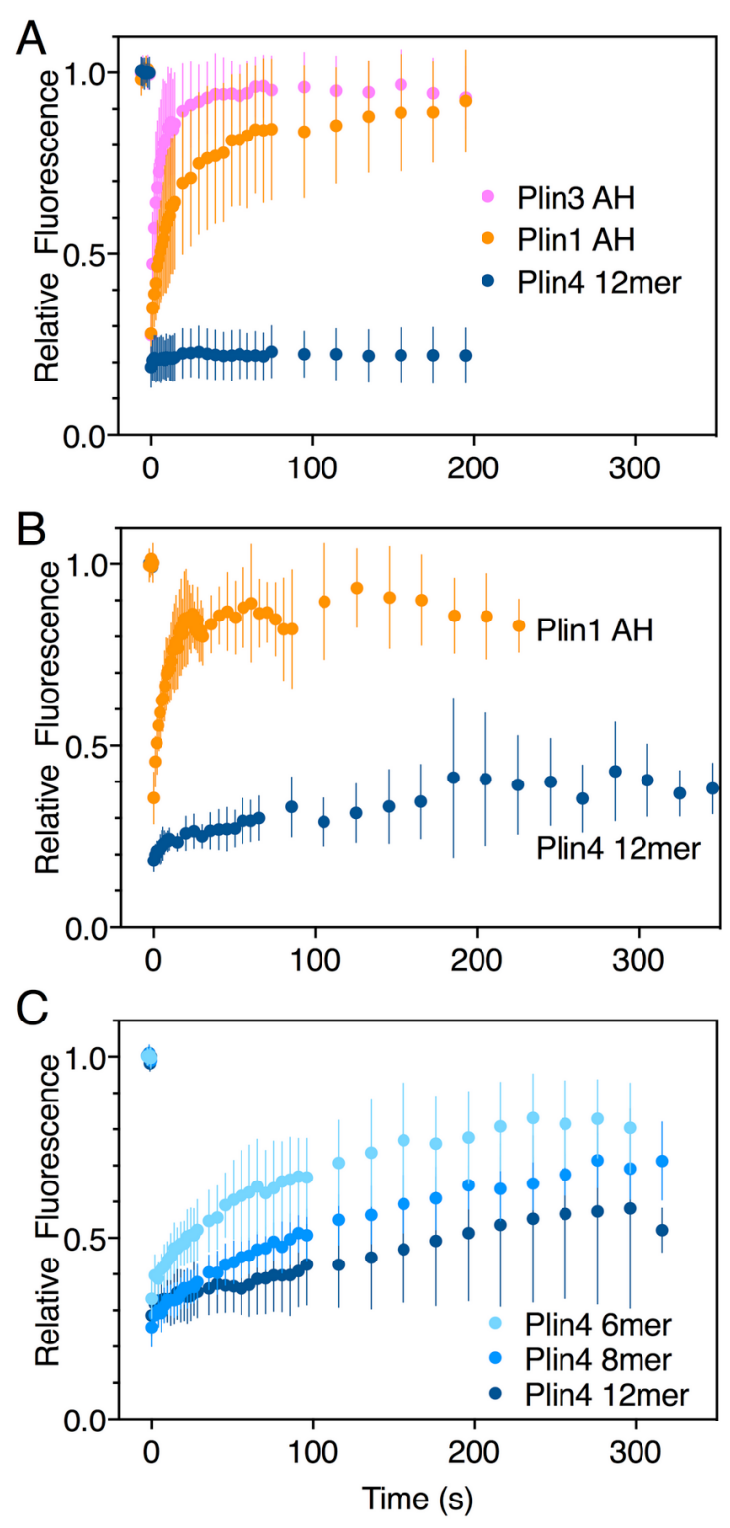

Figure 4. Dynamics of Plin 1, Plin 3, and Plin 4 AHs on LDs or at the plasma membrane in yeast as assessed by FRAP measurement. The AH region of Plin 1, Plin 3 or Plin4 was expressed as a GFP fusion in S. cerevisiae cells. FRAP was performed on LDs or the PM covered with the indicated Plin construct. The graphs show the mean \pm SD of the fluorescence recovery curves from $n$ FRAP measurements on different LDs or different regions of the PM. A. Plin1 AH-GFP ( $n=29)$, Plin3 AH-GFP $(n=36)$ or Plin4 12mer-GFP $(n=24)$ on LDs in pet10 $\Delta+$ OA (growth for 48h, large LDs). B. Plin1 AH-GFP $(n=11)$ or Plin4 12mer-GFP $(n=5)$ on LDs in the yeast strain pet10 $\Delta$ in early stationary cells (small LDs). C. Plin4 6mer-GFP (green; $n=12$ ), Plin4 8mer-GFP (red; $n=7$ ), Plin4 12mer-GFP (blue; $n=7$ ) at the PM in exponentially-growing wild-type cells. 
Figure 5

A

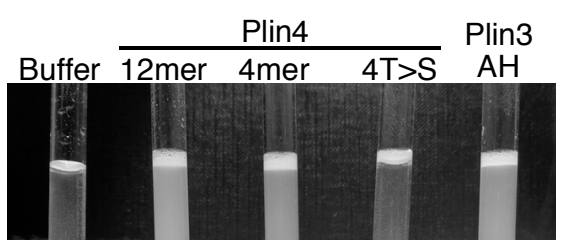

C
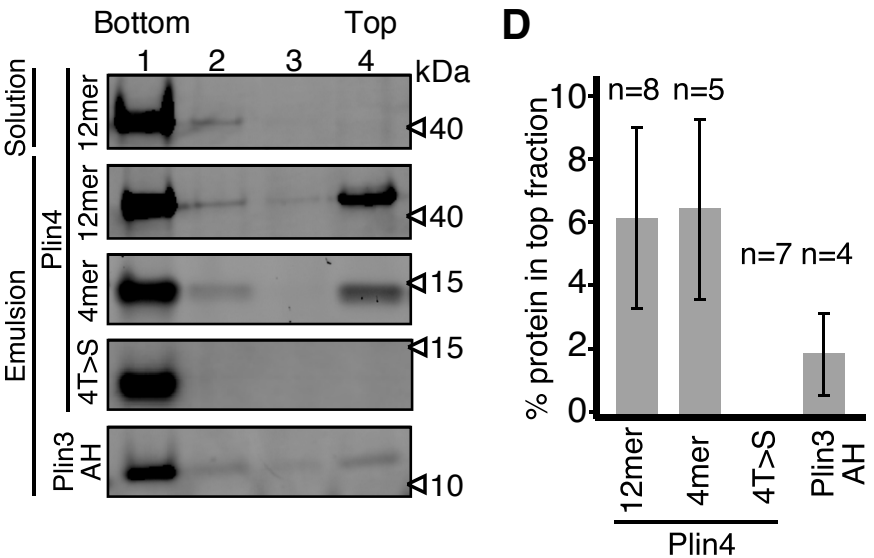

B
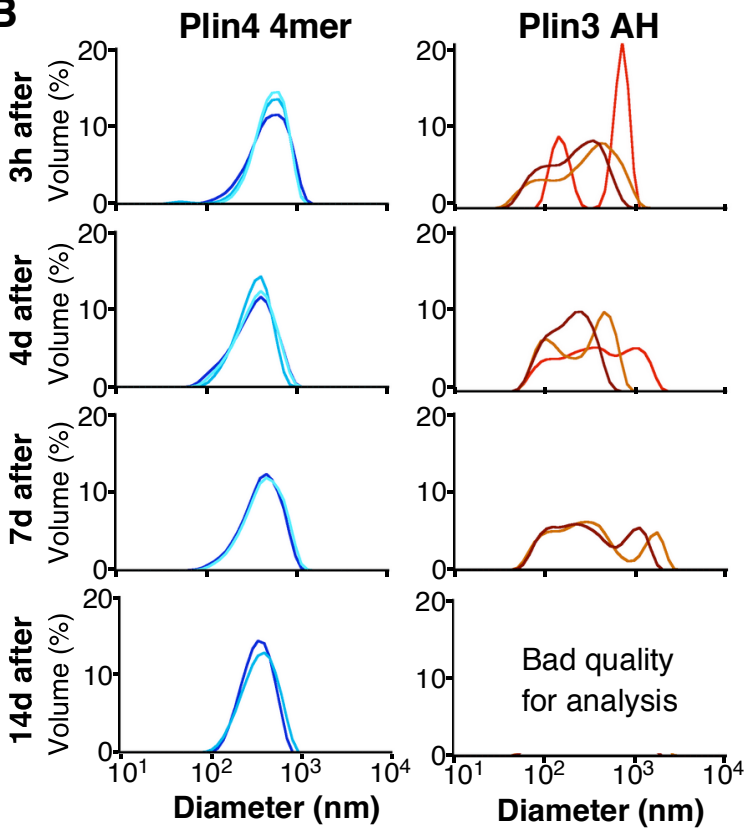

E

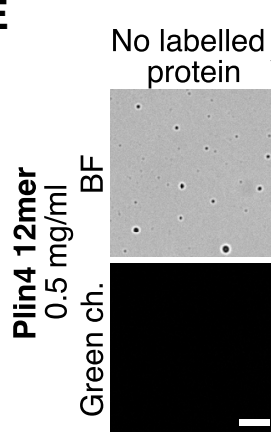

$2 \mathrm{~h}$

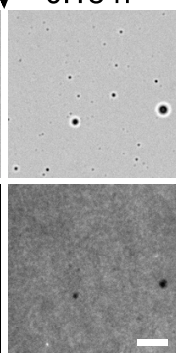

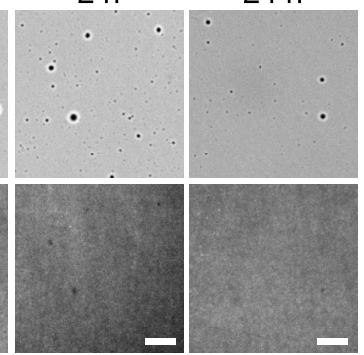

$\mathrm{mg} / \mathrm{m}$
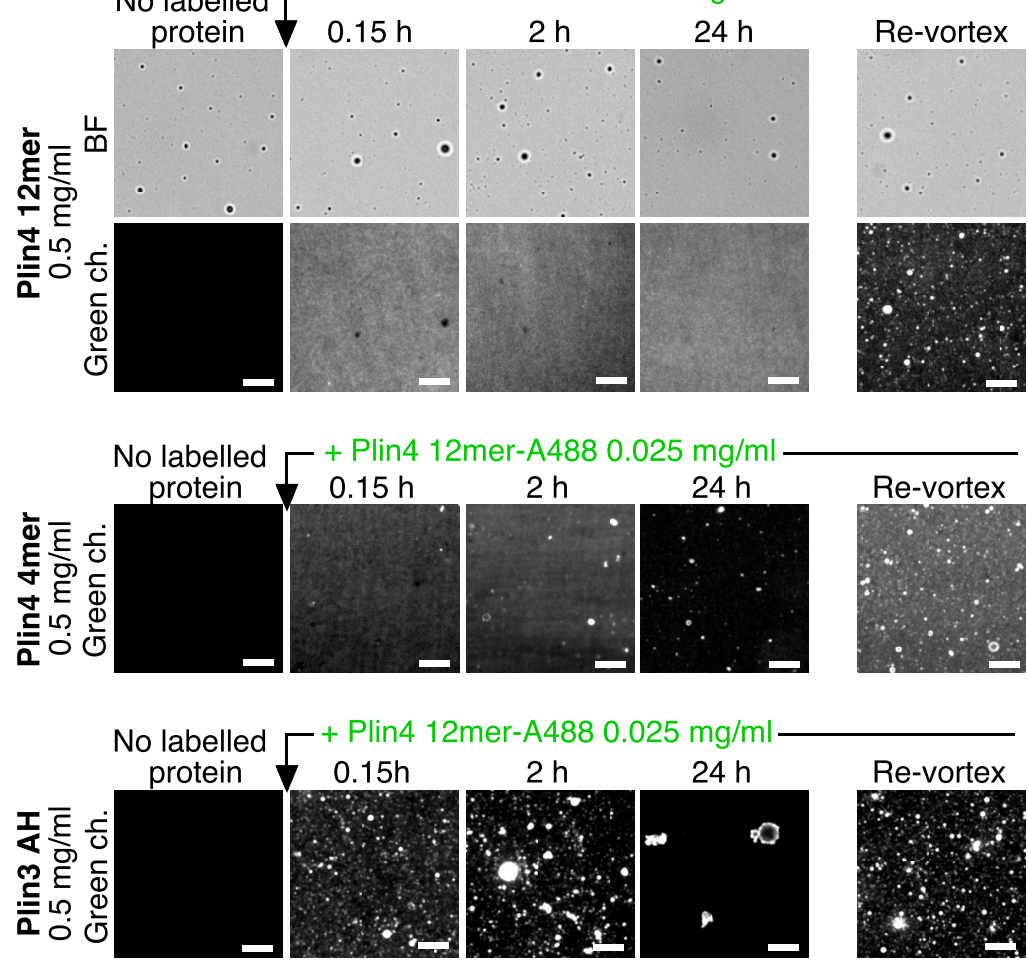

F
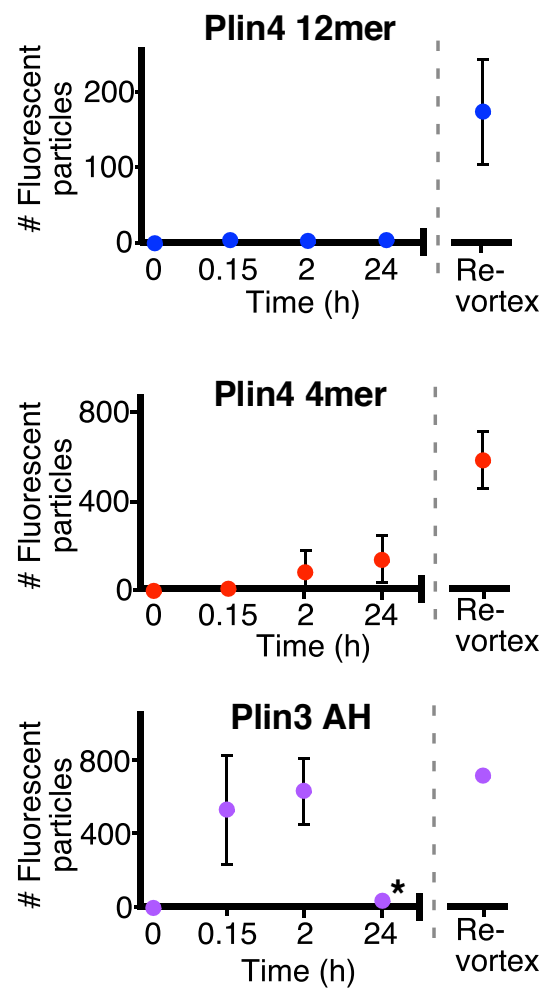
Figure 5. Plin3 AH interacts much less strongly with oil than Plin4 AH. A. Turbidity assays with $0.5 \mathrm{mg} / \mathrm{ml}$ protein solutions of Plin4 $12 \mathrm{mer}$, Plin4 4mer, Plin4 4mer mutant [4T>S], or Plin3 $\mathrm{AH}$ after vigorous vortexing with triolein (15 $\mu \mathrm{l}$ for $285 \mu \mathrm{l}$ of protein solution). B. Size distribution of the droplets in triolein emulsions formed with Plin4 4mer or Plin3 AH at various times after vortexing was determined by DLS. Particle size is shown by volume weighted distributions. Each curve represents one measurement. C. Protein/oil emulsions or protein solutions of the indicated variants of Plin4 or Plin3 AH were mixed with sucrose and loaded on the bottom of a sucrose step gradient. After centrifugation, four fractions were collected from the bottom and equal volumes were analysed by protein gels with Sypro Orange staining. D. Quantification of the experiment shown in C showing the mean \pm SD of protein at the top fraction of the sucrose gradient. The number of repeats for each experiment is indicated above the bar graphs. E. Protein exchange assay in LD emulsions. Top panel: a Plin4 $12 \mathrm{mer}$ $(0.5 \mathrm{mg} / \mathrm{ml}) /$ triolein emulsion was prepared by vortexing. Thereafter, $0.025 \mathrm{mg} / \mathrm{ml}$ Alexa488-labeled Plin4-12 mer was gently added. The emulsion was imaged at the indicated time points by light microscopy in bright field (BF) to see all particles and by fluorescence to detect the coverage of the particles by Alexa-488-labeled Plin4 12 mer. Finally, the suspension was vortexed again to promote maximum incorporation of Alexa488-labeled Plin4 12mer in the emulsion. The middle and lower rows show similar experiments performed with Plin 4 mer and Plin3 AH emulsions, respectively. Scale bars: $5 \mu \mathrm{m}$. F. Quantification of the experiments shown in $\mathrm{E}$. The number of fluorescent particles (mean $\pm \mathrm{SD}$ ) was determined from four separate fields $(73 \times 100 \mu \mathrm{m})$ in the same experiment. The graphs are representative of at least two independent experiments. Time on the $\mathrm{x}$-axis is plotted using logarithmic scale. Asterisks indicates clustering of particles, which resulted a in low total number of fluorescent puncta, as seen in the image. 
A

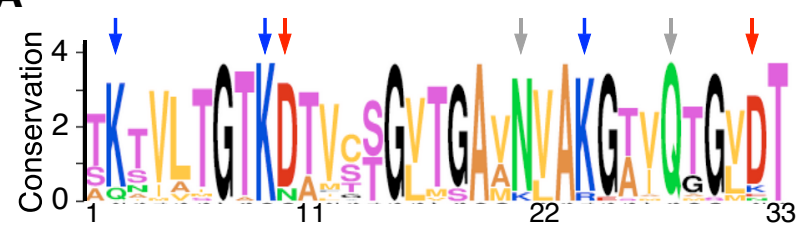

B

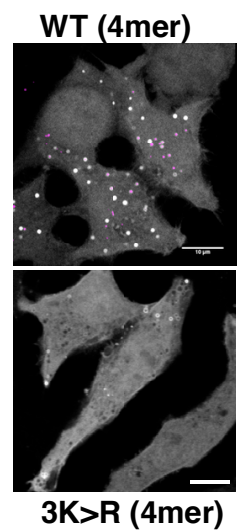

C

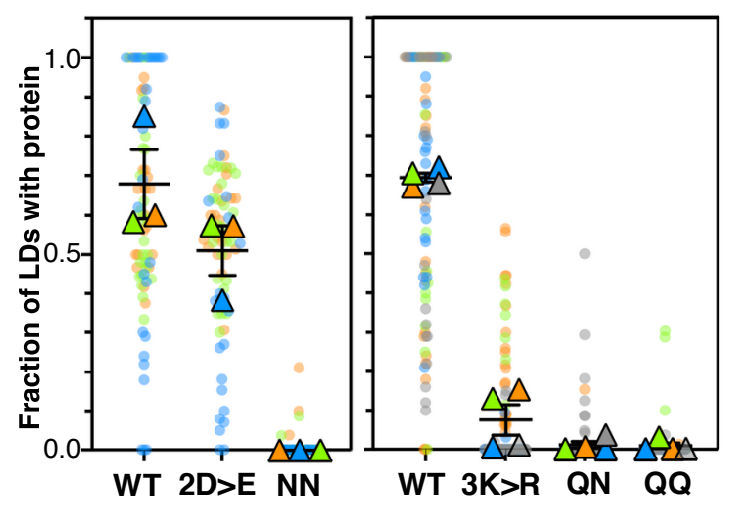

D
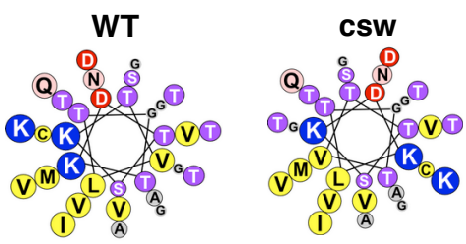

E
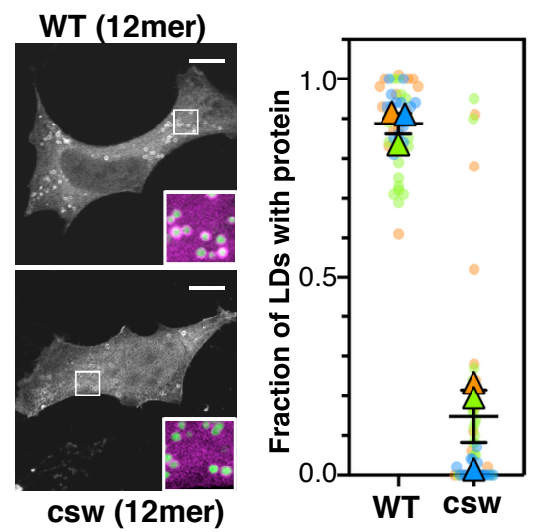
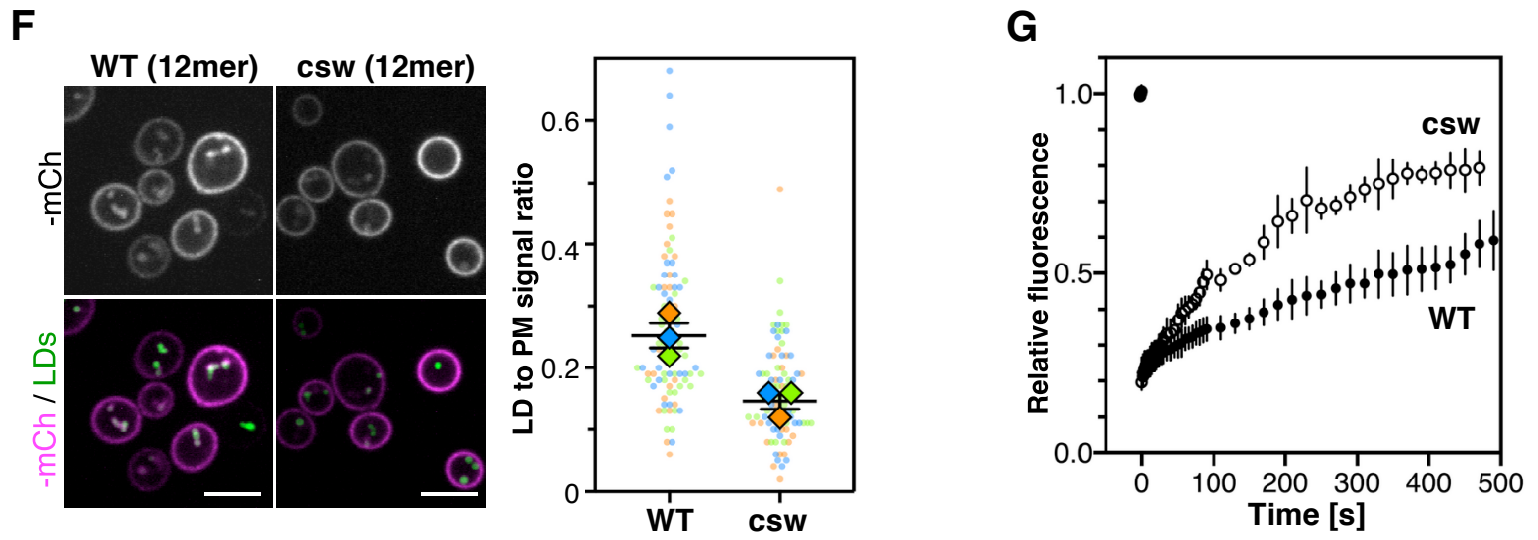

Figure 6.The nature and the distribution of charged residues in the polar face of Plin4 AH contribute is key for specific and stable coating of LDs in cells. A. Weblogo plot of the AH region of human Plin4 as determined by aligning its $2933-$ mer repeats. The vertical arrows indicate the mutated aa: the NQ pair (grey), which was mutated into $N N, Q Q$ or $Q N$; the three $K$ (blue), which were mutated into R, and the two $D$ (red), which were mutated into E. B. Co-localization of GFP-fusion of Plin4 4mer wild-type and 3K>R (in white) with LDs (purple) in HeLa cells. C. Quantification of the percentage of LDs stained with the indicated protein per cell. These "SuperPlots" (Lord et al., 2020) show all data fom 3 to 4 independent experiments, each with a different color; each light dot represents one cell, whereas each triangle shows the mean from one experiment. The black bars show the mean \pm SE of the 3 or 4 independent experiments. D. Helical wheels of Plin4 WT and csw mutant. Mutations were introduced to redistribute positive and negative residues in a symmetrical manner while keeping the number and nature of these residues constant. E. Localization of Plin4 12mer wild-type or csw mutant in HeLa cells. The insets show extended views with the protein in purple and LDs in green (stained with Bodipy). The 'super' plots on the right were built as in C and show the mean \pm SE of the percentage of LDs positive for the indicated protein per HeLa cell as determined from 3 independent experiments. F. Light microscopy images of mCherry fusions of Plin4 12mer wild-type or csw mutant in yeast. Top: mCherry fluorescence $(\mathrm{mCh})$; bottom co-localization of mCherry (purple) with LDs stained with bodipy (green). The relative fluorescence signal of mCherry fusions of Plin4 12mer wild-type or csw mutant on LDs and at the PM in PET10-GFP yeast strain was used to build the SuperPlots shown on the right. Data are from three independent experiments, with $n \geq 25$ for each condition in each assay. G. Fluorescence recovery curves of GFP fusions of Plin4 12mer wild-type or and csw mutant at the PM of late exponential phase yeast cells. 


\section{Figure 7}

A

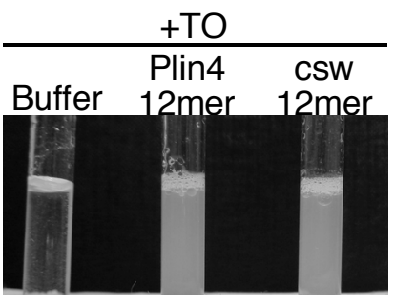

B No labelled

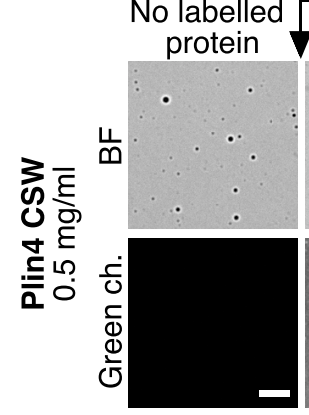

+ Plin4 12
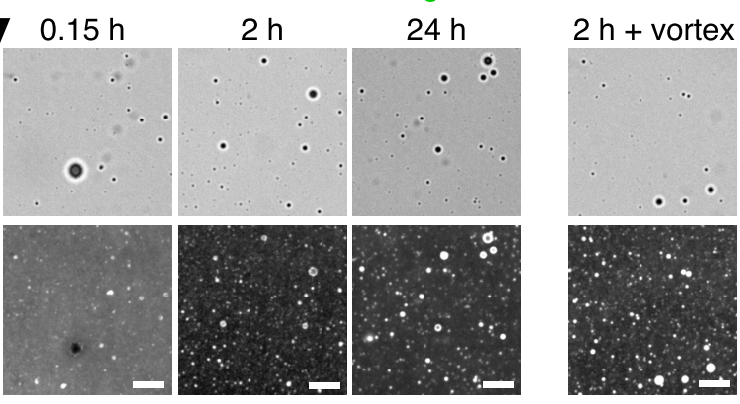

C

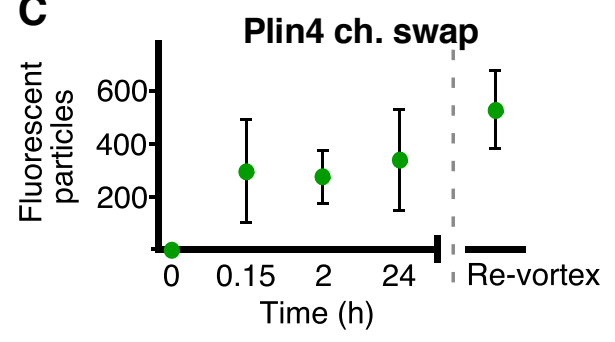

Figure 7. The distribution of charged residues in the polar face of Plin4 AH is key for stable coating of triolein in vitro. A. $15 \mu \mathrm{l}$ triolein was added to $285 \mu \mathrm{l}$ of HK buffer containing Plin4 12mer wild-type or the csw mutant $(0.5 \mathrm{mg} / \mathrm{ml}$ each). After vigorous vortexing, the samples were photographed. B. A triolein emulsion was prepared with Plin4 $12 \mathrm{mer}$ or csw $12 \mathrm{mer}$ mutant $(0.5 \mathrm{mg} / \mathrm{ml}$ protein). At the indicated time, fluorescent Plin4 12mer-Alexa488 $(0.025 \mathrm{mg} / \mathrm{ml})$ was gently added. The emulsions were imaged by light microscopy in the bright field mode (BF) to see all particles and by fluorescence to detect the incorporation of Plin4 12mer-Alexa488 into the proteolipid particles. At the end of the experiment, the suspension was vortexed again to promote maximum incorporation of Plin4 12mer-Alexa488 in the emulsion. C. Quantification of the experiment shown in B. D. Model of how positive and negative charges could selfstabilize different Plin4 AHs at the surface of triolein. 
Figure 8

\# repeats

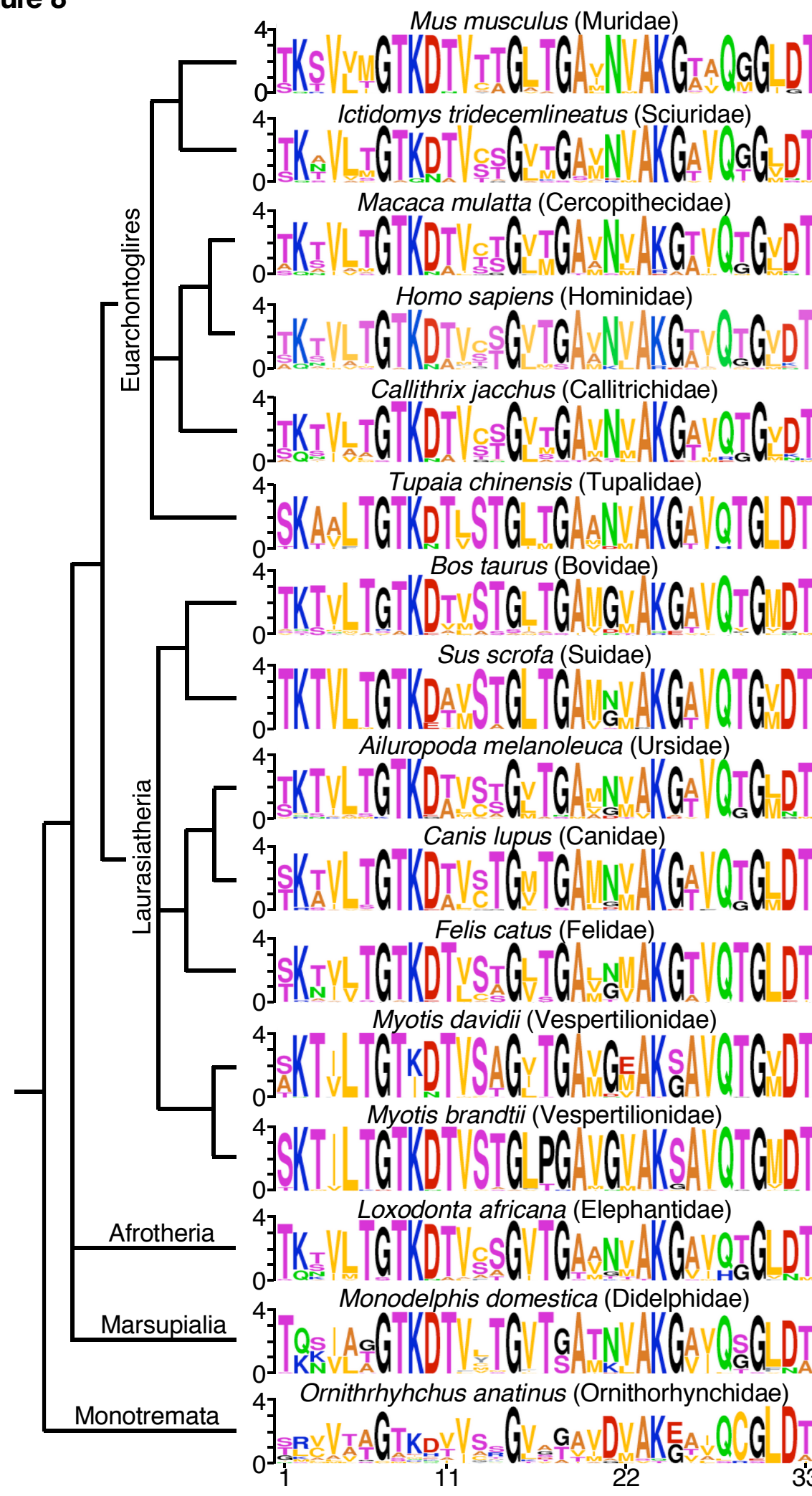

Figure 8. Conservation of 33-aa repeats in Plin4 orthologs in mammalian species. Alignments of the repeats from sixteen Plin4 sequences from diverse mammalian species are shown. The number of repeats identified in each sequence is listed in the right column. Sequences are shown on a phylogenetic tree following (Bininda-Emonds et al., 2007); the main mammalian lineages are indicated on the right side. See Supplementary table 1 for additional information. 